\title{
Survey of Large-Scale MIMO Systems
}

\author{
Kan Zheng, Senior Member, IEEE, Long Zhao, Jie Mei, Bin Shao, \\ Wei Xiang, Senior Member, IEEE, and Lajos Hanzo, Fellow, IEEE
}

\begin{abstract}
The escalating teletraffic growth imposed by the proliferation of smartphones and tablet computers outstrips the capacity increase of wireless communications networks. Furthermore, it results in substantially increased carbon dioxide emissions. As a powerful countermeasure, in the case of full-rank channel matrices, MIMO techniques are potentially capable of linearly increasing the capacity or decreasing the transmit power upon commensurately increasing the number of antennas. Hence, the recent concept of large-scale MIMO (LS-MIMO) systems has attracted substantial research attention and been regarded as a promising technique for next-generation wireless communications networks. Therefore, this paper surveys the state of the art of LS-MIMO systems. First, we discuss the measurement and modeling of LS-MIMO channels. Then, some typical application scenarios are classified and analyzed. Key techniques of both the physical and network layers are also detailed. Finally, we conclude with a range of challenges and future research topics.
\end{abstract}

Index Terms-Large-scale MIMO, 3-D MIMO, channel modeling, physical layer, networking.

$\begin{array}{ll}\text { 2D } & \text { Two-dimensional. } \\ \text { 3D } & \text { Three-dimensional. } \\ \text { 3GPP } & \text { 3rd Generation Partnership Project. } \\ \text { 4G } & \text { Fourth generation. } \\ \text { AA } & \text { Antenna array. } \\ \text { ABS } & \text { Almost blank subframe. } \\ \text { AEs } & \text { Antenna elements. } \\ \text { AoAs } & \text { Azimuth of arrivals. } \\ \text { AoD } & \text { Azimuth of departure. } \\ \text { APS } & \text { Angular power spectrum. } \\ \text { AWGN } & \text { Additive white Gaussian noise. } \\ \text { BBU } & \text { Baseband unit. } \\ \text { BE } & \text { Bandwidth efficiency. } \\ \text { BER } & \text { Bit error ratio. }\end{array}$

Manuscript received September 6, 2014; revised February 26, 2015; accepted April 16, 2015. This work was supported in part by the National 973 Program of China under grant 2012CB316005, the National High Technology Research and Development Program of China under Grant 2014AA01A705, the China Natural Science Funding under Grant 61271183, and the Fundamental Research Funds for the Central Universities under Grant 2014ZD03-02.

K. Zheng, L. Zhao, J. Mei, and B. Shao are with the Wireless Signal Processing and Network Laboratory, Key Laboratory of Universal Wireless Communication, Ministry of Education, Beijing University of Posts and Telecommunications, Beijing 100876, China (e-mail: kzheng@ieee.org)

W. Xiang is with the School of Mechanical and Electrical Engineering, University of Southern Queensland, Toowoomba, Qld. 4350, Australia.

L. Hanzo is with the School of Electronics and Computer Science, University of Southampton, Southampton SO17 1BJ, U.K. (e-mail: 1h@ecs.soton.ac.uk).

Digital Object Identifier 10.1109/COMST.2015.2425294

\begin{tabular}{|c|c|}
\hline $\mathrm{BF}$ & Beamformer. \\
\hline BI-GDFE & $\begin{array}{l}\text { Block-iterative generalized decision feedbac } \\
\text { equalizer. }\end{array}$ \\
\hline CBSM & Correlation-based stochastic model. \\
\hline CEP & Constant envelope precoding. \\
\hline CIRs & Channel impulse responses. \\
\hline CR & Correlation rotation. \\
\hline CRE & Cell range extension. \\
\hline CSI & Channel state information. \\
\hline CSIT & CSI at the transmitter. \\
\hline DASs & Distributed antenna systems. \\
\hline DDCM & Double directional channel model. \\
\hline DoF & Degrees of freedom. \\
\hline DPC & Dirty paper coding. \\
\hline DL & Downlink. \\
\hline DS & Delay spread. \\
\hline $\mathrm{EE}$ & Energy efficiency. \\
\hline eNB & Evolved Node B. \\
\hline EoD & Elevation angle of departure. \\
\hline EoA & Elevation angle of arrival. \\
\hline FD & Frequency domain. \\
\hline FDD & Frequency division duplex. \\
\hline FFR & Fractional frequency reuse. \\
\hline FIR & Finite impulse response. \\
\hline GBSM & Geometry-based stochastic model. \\
\hline HetNet & Heterogeneous network. \\
\hline HomoNet & Homogeneous network. \\
\hline ICI & Inter cell interference. \\
\hline ICIC & Inter-cell interference coordination. \\
\hline i.i.d. & Independent identically distributed. \\
\hline ISD & Inter site distance. \\
\hline IUI & Inter-user interference. \\
\hline LoS & Line of sight. \\
\hline LS-MIMO & Large-scale MIMO. \\
\hline MAC & Media access control. \\
\hline MAP & Maximum posterior probability. \\
\hline MAX-MIN & Maximizing the minimum. \\
\hline MeNB & Macro-cell eNB. \\
\hline MF & Matched filter. \\
\hline MIMO & Multiple-input and multiple-output. \\
\hline MIN-MAX & Minimizing the maximum. \\
\hline ML & Maximum-likelihood. \\
\hline MMSE & Minimum mean square error. \\
\hline MMSE-SIC & MMSE based soft interference cancellation. \\
\hline MRC & Maximum ratio combining. \\
\hline MRT & Maximum ratio transmission. \\
\hline MSE & Mean square error. \\
\hline MUEs & Macro-cell UEs. \\
\hline MU-MIMO & Multi-user MIMO. \\
\hline
\end{tabular}




$\begin{array}{ll}\text { NLoS } & \text { Non line of sight. } \\ \text { OF } & \text { Objective function. } \\ \text { PAs } & \text { Power amplifiers. } \\ \text { PAPR } & \text { Peak to average power ratio. } \\ \text { PBCH } & \text { Physical broadcasting channel. } \\ \text { PDSCH } & \text { Physical downlink shared channel. } \\ \text { PSM } & \text { Parametric stochastic model. } \\ \text { QoS } & \text { Quality of service. } \\ \text { R12 } & \text { Release 12. } \\ \text { RF } & \text { Radio-frequency. } \\ \text { RRU } & \text { Remote radio unit. } \\ \text { RSRP } & \text { Reference signal received power. } \\ \text { RTDD } & \text { Reversed TDD. } \\ \text { RZF } & \text { Regularized ZF. } \\ \text { SDM } & \text { Spatial division multiplexing. } \\ \text { SDMA } & \text { Spatial division multiple access. } \\ \text { SeNBs } & \text { Small-cell eNBs. } \\ \text { SER } & \text { Symbol error ratio. } \\ \text { SF } & \text { Shadow fading. } \\ \text { SFR } & \text { Soft frequency reuse. } \\ \text { SINR } & \text { Signal-to-interference-plus-noise ratio. } \\ \text { SNR } & \text { Signal-to-noise ratio. } \\ \text { SUEs } & \text { Small-cell UEs. } \\ \text { TD } & \text { Time-domain. } \\ \text { TDD } & \text { Time division duplex. } \\ \text { TPC } & \text { Transmit precoding. } \\ \text { TS } & \text { Tabu search. } \\ \text { UEs } & \text { User terminals. } \\ \text { UL } & \text { Uplink. } \\ \text { VP } & \text { Vector perturbation. } \\ \text { VRM } & \text { Virtual ray model. } \\ \text { ZF } & \text { Zero-forcing. } \\ & \end{array}$

\section{INTRODUCTION}

W ITH the evolution of smart terminals and their applications, the need for multimedia services rapidly increases recently. Thus, the capacity of wireless communications networks has to be increased in order to guarantee the Quality of Service (QoS) requirements of mobile applications. Meanwhile, telecommunication manufactures and operators have also foreseen that the load of wireless communications networks is increasing exponentially [1]. Therefore, it is necessary to introduce new technologies to meet the demands of explosive traffic for next-generation wireless communications networks.

Bandwidth Efficiency (BE) is usually one of the most important metrics to select candidate technologies for next-generation wireless communications systems. Meanwhile, with excessive power consumption in wireless communications networks, both carbon emissions and operator expenditure increase year by year [2], [3]. As a result, Energy Efficiency (EE) has become another significant metric for evaluating the performances of wireless communications systems with some given BE constraints [4]-[6].

Multiple-Input and Multiple-Output (MIMO) technology has attracted much attention in wireless communications, because it offers significant increases in data throughput and link range without an additional increase in bandwidth or transmit power. In 1993 and 1994, a MIMO approach was proposed and the corresponding patent was issued [7], where multiple transmit antennas are co-located at one transmitter with the objective of improving the attainable link throughput. Then, the first laboratory prototype of spatial multiplexing was implemented to demonstrate the practical feasibility of MIMO technology [8]. Nowadays, MIMO has been accepted as one of key technologies in the Fourth Generation (4G) wireless communications systems. When an evolved Node B (eNB) equipped with multiple antennas communicates with several User Terminals (UEs) at the same time-frequency resources, it is referred to as Multi-User MIMO (MU-MIMO). MU-MIMO is capable of improving either the $\mathrm{BE}$ or the reliability by improving either the multiplexing gains or diversity gains [9]. In order to scale up these gains, the Large-Scale MIMO (LS-MIMO) concept, which is also known as massive MIMO scheme often also associated with the terminologies of large-scale antenna systems, very large MIMO, very large multi-user-MIMO, fulldimensional MIMO, hyper MIMO, etc, was proposed by Marzetta in [10]. More explicitly, a LS-MIMO refers to the system that uses hundreds of antennas to simultaneously serve dozens of UEs. Both theoretical and measurement results indicate that a LS-MIMO is capable of significantly improving the $\mathrm{BE}$, which simultaneously reducing the transmit power [11], [12]. As a result, a LS-MIMO is regarded as a candidate technique for next-generation wireless communications systems conceived for the sake of improving both their BE and EE.

As the down tilt of an Antenna Array (AA) is fixed, traditional MIMO technology can only adjust signal transmission in the horizontal dimension. In order to exploit the vertical dimension of signal propagation, AAs, such as rectangular, spherical and cylindrical AAs, were studied by the 3rd Generation Partnership Project (3GPP) [13]-[15]. MIMO with these arrays can adjust both azimuth and elevation angles, and propagate signals in Three-Dimensional (3D) space, thus termed 3D MIMO. To further increase capacity, 3D MIMO deploys more antennas to achieve larger multiplexing gains. Meanwhile, LS-MIMO adopts rectangular, spherical or cylindrical AAs in practical systems considering the space of AAs. Therefore, 3D MIMO with massive antennas can be seen as a practical deployment means of LS-MIMO, and both of them are investigated in this paper.

LS-MIMO can improve BE since it can achieve large multiplexing gains when serving tens of UEs simultaneously [10], [16]. The significant increase in EE is due to the fact that the use of more antennas helps focus energy with an extremely narrow beam on small regions where the UEs are located [17]. Apart from these advantages, LS-MIMO can enhance transmission reliability owing to the excessive Degrees of Freedom (DoF) [18]. Inter-User Interference (IUI) can also be alleviated because of the extreme narrow beam [11]. In an LS-MIMO system, individual element failure of the AA is not detrimental to the performance of the entire system [11]. Simple low-complexity signal processing algorithms are capable of approximating the performance achieved by optimal methods, such as MaximumLikelihood (ML) multiuser detection and Dirty Paper Coding (DPC) [12]. The latency of the air interface can be reduced and 
TABLE I

CuRrent RESEARCH DiRECTIONS OF LS-MIMO

\begin{tabular}{|c|c|c|c|}
\hline Topics & Description & [11], [12], [21] & This paper \\
\hline AA configuration & Rational antennas deployment & $x$ & $\sqrt{ }$ \\
\hline Channel model & Channel measurement and modeling & $\sqrt{ }$ & $\sqrt{ }$ \\
\hline Channel estimation & Efficient estimation algorithms for pilot reuse & $\sqrt{ }$ & $\sqrt{ }$ \\
\hline Precoding and detection & Low-complexity and efficient precoder and detector & $\sqrt{ }$ & $\sqrt{ }$ \\
\hline Performance and limitation & BE, EE and reliability performance and limitation factors & $\sqrt{ }$ & $\sqrt{ }$ \\
\hline Practical applications & Network deployment and optimization & $\times$ & $\sqrt{ }$ \\
\hline Resource management & Management of frequency, time and spatial resources & $x$ & $\sqrt{ }$ \\
\hline Interference controlling & Pilot contamination alleviation and interference suppression & $\sqrt{ }$ & $\sqrt{ }$ \\
\hline Massive distributed antennas & Design and analysis of distributed antennas in indoor and outdoor environments & $x$ & $\sqrt{ }$ \\
\hline
\end{tabular}

the protocols at the Media Access Control (MAC) layer can be simplified because of the channel harden phenomenon and sufficient capacity [19].

Certainly, the complexity of signal processing, including Transmit Precoding (TPC), channel estimation and detection, increases with the number of antennas. On the other hand, the maximum number of orthogonal pilot sequences is limited by the coherence interval and coherence bandwidth. Therefore, the performance of LS-MIMO systems is constrained by pilot contamination due to pilot reuse in multi-cell scenarios [10]. Moreover, compared to the Physical Downlink Shared Channel $(\mathrm{PDSCH})$ employing either precoding or beamforming, the Signal-to-Interference-plus-Noise Ratio (SINR) of the Physical Broadcasting Channel (PBCH) is lower due to the omnidirectional signal transmission [20].

Currently, fundamental theoretical problems and several physical layer techniques of LS-MIMO have been already widely investigated. For example, the capacity and the realistic performance of precoding and detection for LS-MIMO have been analyzed from the viewpoint of information theory [12], [21]. Additionally, the advantages and disadvantages, the potential applications and limitations of LS-MIMO were generally given in [11]. However, the performance of LS-MIMO is much more affected by practical factors, which are not well summarized so far. Therefore, besides a comprehensive investigation on the theoretical performance of LS-MIMO, this paper pays more attention to the discussions of issues from the system point of view, such as practical channel models under different AAs, practical application scenarios for LS-MIMO, networking techniques, and so on. According to the current literature related to LS-MIMO, the major research directions about LS-MIMO are listed in Table I, some of which have been investigated in [11], [12] and [21] while others are not.

The remainder of this paper is organized as shown in Fig. 1. The measurement and modeling of LS-MIMO channels are discussed in Section II. Section III introduces main scenarios and applications in wireless communications networks. The theoretical and measured performances of LS-MIMO with different precoders and detectors are discussed for both single-cell and multi-cell scenarios in consideration of perfect and imperfect Channel State Information (CSI) in Section IV. Section V investigates related networking techniques, such as Inter-Cell Interference Coordination (ICIC) and scheduling. Section VI

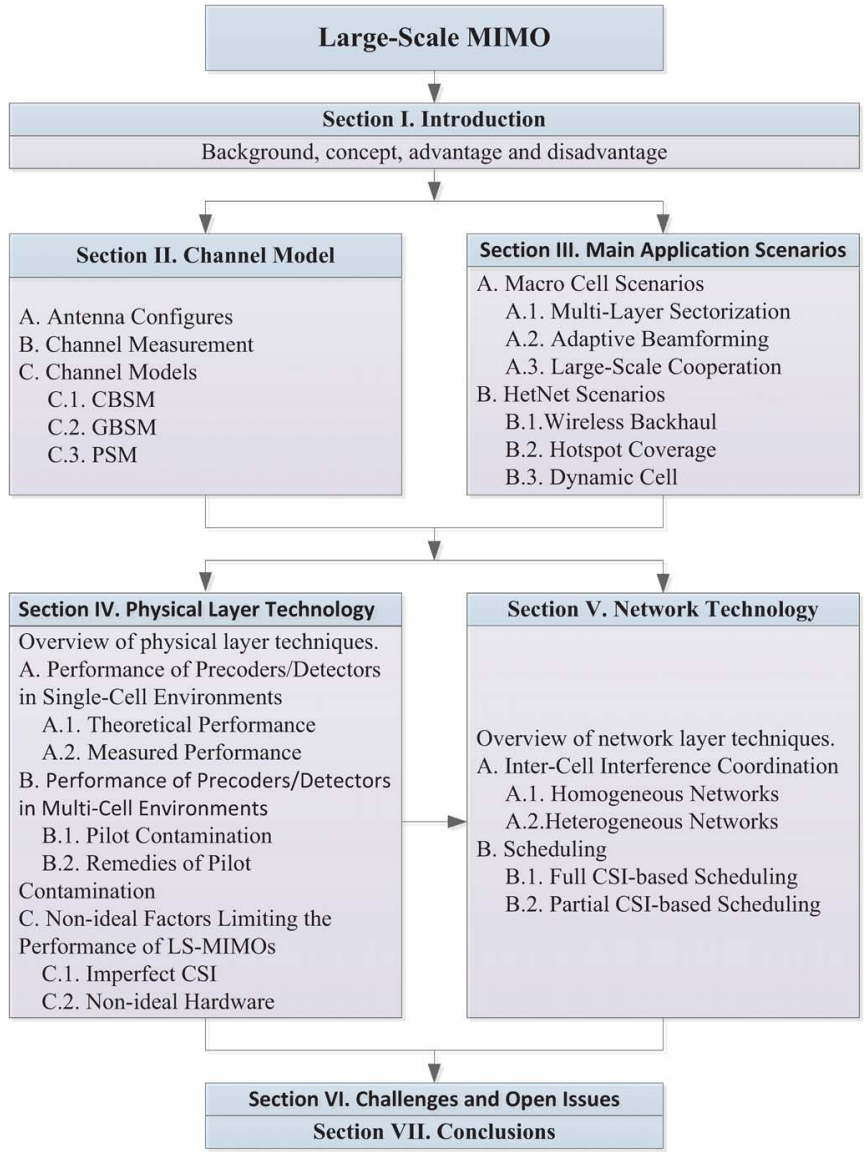

Fig. 1. Structure of this survey paper.

identifies challenges and research directions, and Section VII concludes this paper.

\section{Channel Model of LS-MiMO}

Channel modeling is a fundamental problem in the sense of evaluating the performance of the LS-MIMO system. In this section, we first introduce several typical antenna configurations design for LS-MIMO. Then, the main properties of LS-MIMO channels are characterized with the aid of measurement results. Next, three types of channel models are presented, 


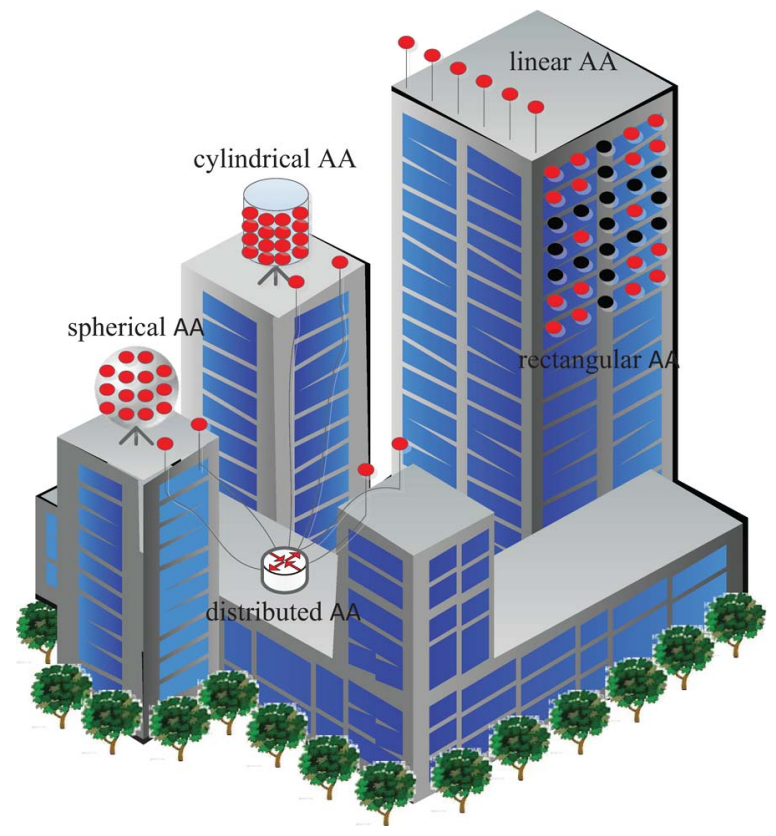

Fig. 2. Various antenna configurations.

which are suitable for either theoretical analysis or practical evaluation.

\section{A. Antenna Configurations}

In a traditional passive AA, the Radio-Frequency (RF) circuit is usually connected to its physical antennas through an RF cable. In order to reduce the loss imposed by the RF cable and to save the costs of installation and maintenance, a Remote Radio Unit (RRU) in conjunction with a Baseband Unit (BBU) has become a preferred configuration recently [22]. The baseband digital signal generated by the BBU is sent to the RRUs through an optical fiber. The RF circuit is placed as close as possible to the physical AA. Furthermore, active AAs operating without RF cables are now also commercially available, which enabling engineers to carefully configure a LS AA [23]. In an active $\mathrm{AA}$, the RF circuit and the AA are integrated into a single circuit board, which is an important milestone in the development of AA.

Fig. 2 illustrates several typical LS AAs, namely the linear AA, spherical AA, cylindrical AA, rectangular AA, and distributed AA [11]. The linear AA is an example of TwoDimensional (2D) AAs, whereas the spherical AA, cylindrical AA and rectangular AAs belong to the family of 3D AAs. Considering the space limitations at both the eNBs and UEs, the spherical, cylindrical and rectangular AAs are more realistic for practical systems. The distributed AA is mainly used either inside buildings or for outdoor cooperation, and the linear AA is mostly assumed in theoretical analysis and realistic measurements.

Moreover, due to the associated aspects of aesthetics and potential health issues, commercial deployments of LS AAs have been partially opposed both by the public and by the organizations. By integrating the AEs into the environment, LS AAs can be rendered virtually invisible. An aesthetically pleasing method is to deploy LS AA as part of the building's facade or signage in an irregular fashion [20], e.g. the black AEs of a rectangular AA may assume the shape of the Chinese character "Zhong," as shown in Fig. 2. On the other hand, in order to reduce the side lobes of the irregular AA, advanced algorithms relying on subarrays [24], on orthogonal placement [25], or on parasitic AAs [26] can be introduced for improving the beamforming performance of these irregular AAs.

\section{B. Channel Measurements}

Realistic channel measurements have been carried out in [27], [28] in an effort to identify the main characteristics of LS-MIMO channels. As shown in Table II, different antenna configurations may be considered under different scenarios at a carrier frequency of $2.6 \mathrm{GHz}$. The outdoor measurements in [27] focus mainly on the impact of the number of antennas imposed on the small-scale fading characteristics. When a linear AA is employed at the eNB, both the non-stationary nature of the fading and the near-field AA effects have been studied in order to capture the main properties of a realistic channel model [27]. However, it requires further investigations to ascertain whether these properties are valid for both spherical as well as cylindrical and rectangular arrays.

The main results of these measurements may be summarized as follows:

- Since different Antenna Elements (AEs) of the AA at the eNB may encounter different multi-path clusters and the AA is also often subjected to shadow fading, the accurate modeling of LS-MIMO systems in practical nonstationary propagation scenarios remains to a large extent an open challenge [27];

- The Channel Impulse Responses (CIRs) experienced by UEs become more de-correlated from each other in the case of large AAs, because having more AEs allows one to more accurately distinguish both their CIRs and their angles of arrival [29], [30]. In other words, having more AEs at the eNB is capable of achieving improved orthogonality amongst different UEs in comparison to their traditional small-scale MIMO counterparts. It is particularly important in Spatial Division Multiplexing (SDM) or Spatial Division Multiple Access (SDMA) systems, where the unique and user-specific CIRs are used for distinguishing the UEs and the transmission streams; and

- The linear AA has the better angular resolution in azimuth than the cylindrical array. However, the latter is capable of achieving a beneficial resolution in both azimuth and elevation, which may be more useful in high-rise urban environments [28].

\section{Channel Model}

Typically, three types of channel models have been used for evaluating the performance of wireless communications systems, namely the Correlation-Based Stochastic Model (CBSM), the Parametric Stochastic Model (PSM) and the GeometryBased Stochastic Model (GBSM) [31], [32]. The complexity of the CBSM is low so that it is mainly used for evaluating 
TABLE II

Channel Measurements

\begin{tabular}{|l|r|r|r|r|}
\hline Scenario & Antenna configuration & Antenna space & Bandwidth & Measured metrics \\
\hline Outdoor [27] & Linear array with 128-antenna & Half a wavelength & $50 \mathrm{MHz}$ & $\begin{array}{c}\text { Channel gain, } K \text {-factor, Angular Power Spectrum (APS), } \\
\text { eigenvalue distribution, correlation of antennas and UEs }\end{array}$ \\
\hline $\begin{array}{l}\text { Indoor to } \\
\text { outdoor [29] }\end{array}$ & $\begin{array}{r}\text { 32-antenna rectangular to } \\
\text { 128-antenna cylindrical array }\end{array}$ & Half a wavelength & $50 \mathrm{MHz}$ & Correlation \\
\hline Outdoor [28] & $\begin{array}{r}\text { Cylindrical and linear } \\
\text { array with 128-antenna }\end{array}$ & Half a wavelength & $50 \mathrm{MHz}$ & Large-scale fading and angular resolution \\
\hline Outdoor [30] & Virtual array with 112-antenna & - & $20 \mathrm{MHz}$ & Correlation, inverse condition number \\
\hline
\end{tabular}

the theoretical performance of MIMO systems. However, it is somewhat simplistic and hence inaccurate for a realistic MIMO system. Therefore, it is not directly applicable to the modeling of wireless channels, when encountering a spherical wavefront. By contrast, the GBSM model is capable of accurately describing the realistic channel properties, and hence it is more suitable for LS-MIMO channels, albeit with an increased computational complexity. The complexity of the PSM tends to be higher than that of the CBSM, while the accuracy of the PSM is lower than that of the GBSM, which results in paucity of studies on the PSM in LS-MIMO systems. Therefore, in this section, we mainly consider the CBSM in the context of theoretical analysis and the GBSM for realistic performance evaluation, but we also aim for shedding some light on the PSMs designed for LS-MIMOs.

1) CBSM: There are three kinds of simplified CBSMs, i.e., the non-dispersive independent identically distributed (i.i.d.) Rayleigh fading model, the non-dispersive correlated channel model, and the dispersive multi-path channel model, where each tap is modeled as either a correlated or uncorrelated fading process.

- Non-dispersive i.i.d. Rayleigh channel model: When an i.i.d. Rayleigh fading channel is assumed for an LS-MIMO system, no correlation exists between the transmit and receive antennas. Hence, the elements of the fast fading matrix are i.i.d. Gaussian variables;

- Non-dispersive correlated Rayleigh channel model: In order to characterize the Doppler-induced received signal correlation, the correlated channel model has been considered for characterizing the achievable performance of LS-MIMO systems [16]. The fast fading matrix of the correlated channel model is formed by the product of the correlation matrix and the standard complex-valued Gaussian matrix. The correlation matrix quantifies the long-term correlation of the AEs at both the transmitter and receiver, which can be acquired by measurements. By contrast, the complex-valued Gaussian matrix describes the i.i.d. Rayleigh fading channel; and

- Dispersive multi-path channel model: The dispersive multi-path channel model of LS-MIMO systems can have different distributions of the Azimuth of Arrivals (AoAs) from different UEs [33]. In this model, each UE's CIR is constituted by multiple independent paths arriving from different directions. Each independent path is characterized by a path attenuation multiplied by the steering vector of an AoA. When UEs are located at different angular positions, they can be separated according to their AoAs. Therefore, this model is useful in analyzing the performances of the IUI or Inter Cell Interference (ICI) schemes.

2) GBSM: According to the modeling of scattering propagation environments, the GBSM can be classified into singlering [34], twin-ring [35] and elliptical models [36]. However, depending on whether the elevation angle is considered or not, the GBSM involved for an LS-MIMO system is mainly used in the context of elliptical models and can be divided into 2D and $3 \mathrm{D}$ channel models. When an AA is employed at the eNB, the angle of elevation is fixed and the 2D channel model is adequate for accurately evaluating the performance. However, if a practical spherical, cylindrical or rectangular AA is adopted at the eNB, the 3D channel model with an adjustable angle of elevation has to be considered.

- 2D channel model: When adopting a linear AA, a nonstationary spherical wavefront has been observed by LS-MIMO channel measurements [27], [28]. Similarly, a non-stationary wavefront has also been observed for a linear 128-element AA in a semi-urban area [37]. However, due to the fact that the non-stationary spherical wavefront critically affects both the receiver design and its performance, it is important to model this property of the LS-MIMO channel. So, the COST 2100 channel model of [38] has been extended to include the effect of the wavefront's non-stationary [37];

When considering both the non-stationary wavefront propagation phenomenon and the spherical nature of the waveform, an elliptical GBSM is proposed for LS-MIMO systems based on a linear AA in [39], [40]. Also, the birthdeath process of clusters appearing and disappearing is discussed in [39] for the sake of characterizing its nonstationary nature. The results in [39] show that the phases of the linear AA responses are no longer linear, and the AoAs impinging on the AA gradually shift, in agreement with the measurement results in [27]; and

- 3D channel model: The model methodology and parameters of some existing models, such as the 3D channel model of the WINNER+ project [41], depend primarily on literatures, rather than on realistic measurements [42]. Thus, it needs further verification by realistic channel measurements, including the elevation characteristics of 


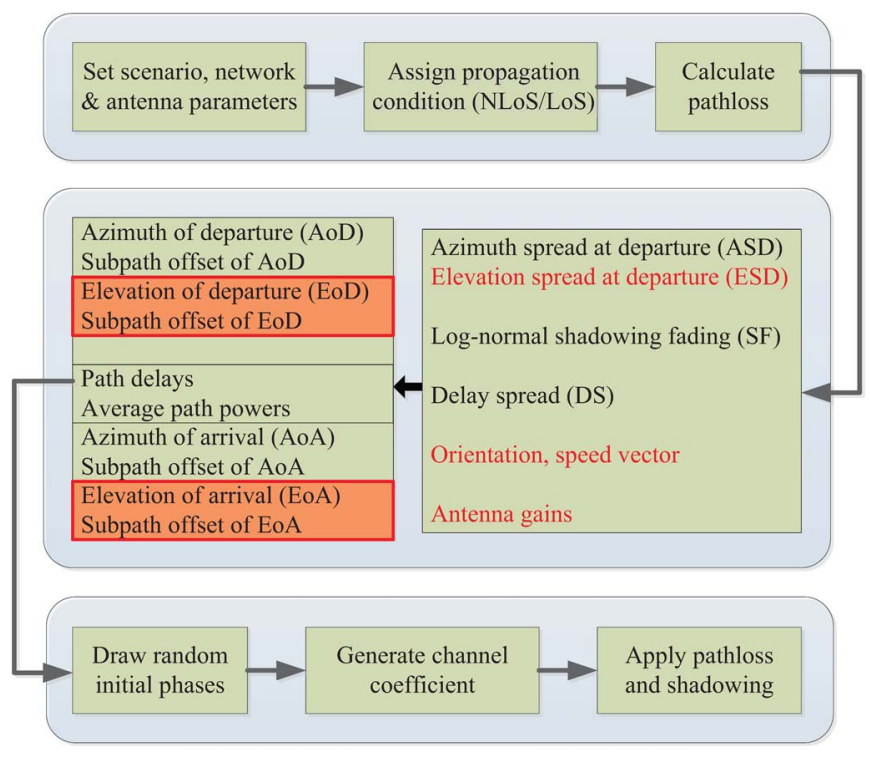

Fig. 3. Procedures of 3D channel modeling.

the $\mathrm{AA}$ as well as the cross-correlation matrix of the large-scale fading parameters. Therefore, the existing 3D channel models cannot be directly applied in realistic scenarios.

In the WINNER II, WINNER+ and COST273 projects, the procedures of modeling the 3D MIMO channel have been proposed as detailed in Fig. 3 [43], [44]. The main parameters of the 3D channel model consist of the Shadow Fading (SF), the Delay Spread (DS), the Ricean $K$-factor, the AoA, Azimuth of Departure (AoD), the Elevation angle of Arrival (EoA) and the Elevation angle of Departure (EoD). The EoA and EoD are of particular interest during the modeling. Moreover, the cross correlation matrix of the channel has been extended from a 5-dimensional to a 7-dimensional matrix, and the correlations between any two parameters have also been characterized in detail.

In 3GPP Release 12 (R12) [44], there are three scenarios at present, namely the urban micro cell associated with a high UE density, the urban macro-cell having a high UE density and the urban macro cell associated with one high-rise AA per sector and the Inter Site Distance (ISD) of 300 meter. The distribution of the related parameters and the cross correlation matrix of the 3D channel model associated with a rectangular AA have been measured, and documented in [44]. According to the procedures of modeling the 3D MIMO channel [44], realistic channel coefficients can be generated for characterizing the abovementioned three scenarios. However, the non-stationary nature of LS-MIMO channels has not been taken into account in [28], [44]. Therefore, the properties and the modeling of the 3D MIMO channel requires further study.

3) PSM: The PSM describes the signals impinging on the receivers as a superposition of waves. A common form of these models employs the structure of a tapped delay line, where each tap reflects a specific propagation path. There are some PSMs for traditional MIMO, such as the Double Directional Channel Model (DDCM) of [45] and the Virtual Ray Model (VRM) of [46], where different methods are used for modeling each tap of the channel. But again, the PSMs are less well studied in the context of LS-MIMOs since they are more complex in terms of their theoretical analysis than the CBSMs. Nevertheless, they constitute viable design alternatives, which are capable of reducing the complexity of the GBSMs for LS-MIMOs. Therefore, the development of the PSM for LS-MIMO systems requires further research efforts, before it becomes a reality.

\section{Summary of LS-MIMO Channels}

The antenna configuration directly affects the characteristics of an LS-MIMO channel. The linear AA gives rise both to non-stationary channel characteristics and to near-field effects, while the rectangular, spherical and cylindrical AAs are capable of accurately directing the beam propagation in the 3D space. Therefore, the choice of the configuration of an AA conceived for particular scenarios requires further investigations.

Currently, the CBSMs are mainly used for analyzing the theoretical performance of LS-MIMO systems attributed to its simplicity. Measurements have also been conducted for validating the accuracy of this model. The non-stationary LS-MIMO channel and the spherical wave propagation effects have been regarded as the in-built properties of the linear AA. The channel model reflecting both the non-stationary LS-MIMO propagation phenomenon and the spherical wave effect propagation effects has been established for the linear AA, which relies on a cluster-based model. Furthermore, an improved 3D channel model has been specified by the 3GPP. However, characterizing the non-stationary propagation for the spherical, cylindrical and rectangular arrays requires further measurements. In conclusion, how to accurately model the channel of LS-MIMOs still remains an open problem to a large extent.

\section{Main Application Scenarios}

This section presents a set of application scenarios, which capture the major dynamics that are of interest in LS-MIMO systems. As an essential step of the study, the definition of application scenarios may present a guide for developing key techniques. As shown in Table III, all of these scenarios under different network deployments can be roughly classified into two types, i.e., Case 1 Homogeneous Network (HomoNet) with only macro-cell deployment and Case 2 Heterogeneous Network (HetNet) with both macro-cell and small cells. Next, more details of these scenarios are discussed.

\section{A. Case 1: Homogeneous Network Scenarios}

1) Case 1A-Multi-Layer Sectorization: Upon increasing the number of UEs and their carried tele-traffic in urban environments, increased system capacity is required for supporting customer requirements. Traditionally, sectorization techniques are used for providing services to a growing population, which simply divide a cell into multiple sectors, thus increasing network capacity. The equipment costs can also be reduced by 
TABLE III

TYPICAL APPLICATION SCENARIOS

\begin{tabular}{|c|r|r|r|}
\hline \multirow{4}{*}{ HomoNet } & Type & Description & Feature \\
\cline { 2 - 5 } & Case 1A & Multi-layer sectorization & $\begin{array}{r}\text { Easy to implement } \\
\text { High multiplexing gains }\end{array}$ \\
\cline { 2 - 4 } & Case 1B & Adaptive beamforming & $\begin{array}{r}\text { Narrow beam and little interference } \\
\text { Flexible with elevation and azimuth }\end{array}$ \\
\cline { 2 - 5 } HetNet & Case 1C & Large-scale cooperation & $\begin{array}{r}\text { Coverage enhancement } \\
\text { Efficient cooperation }\end{array}$ \\
\cline { 2 - 4 } & Case 2A & Wireless backhaul & $\begin{array}{r}\text { Low infrastructure costs } \\
\text { Flexible and extendible }\end{array}$ \\
\cline { 2 - 4 } & Case 2B & Hotspot coverage & $\begin{array}{r}\text { High throughput } \\
\text { Superior elevation resolution }\end{array}$ \\
\cline { 2 - 4 } & Case 2C & Dynamic cell & $\begin{array}{r}\text { Adjusting cell adaptively } \\
\text { Balancing network load }\end{array}$ \\
\hline
\end{tabular}

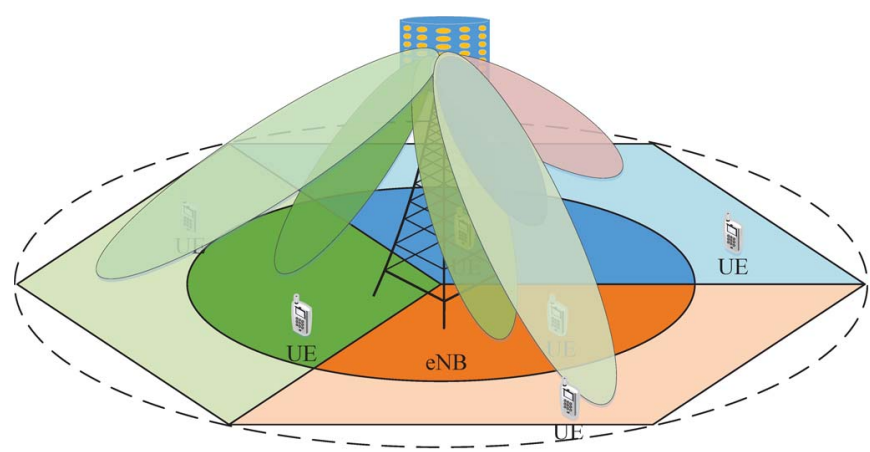

Fig. 4. Illustration of Case 1A: Multi-layer sectorization.

allowing a single eNB to serve either three $120^{\circ}$ sectors or six $60^{\circ}$ sectors. However, although sectorization is capable of improving the area $\mathrm{BE}$, this benefit comes at the expense of a potentially increased interference among sectors due to nonideal sector-antenna patterns. Therefore, more efficient techniques are required to further increase the achievable network capacity.

As illustrated in Fig. 4, accurate sectorization in LS-MIMO systems can be achieved by high-selectivity angular beamforming performed horizontally, which is capable of reducing the interference among sectors. Moreover, the coverage of each beam can be changed by adjusting the elevation angle of 3D beamforming. By this way, a conventional fixed sector can be further spitted into inner and outer sectors, each of which can be served by a 3D Beamformer (BF) with the same horizontal but different elevation angles. The same frequency radio resources are reused by all the sectors, which is capable of significantly increasing the number of UEs served and/or of improving the network's throughput.

2) Case 1B-Adaptive Beamforming: Fixed BFs are so called because the weights that multiply the signals at each element of the AA remain unchanged during operation. By contrast, the weights of an adaptive $\mathrm{BF}$ are continuously updated based on the received signals in order to suppress spatial interference, e.g., as depicted in Fig. 5. This process may be carried out in either the Time-Domain (TD) or Frequency Domain (FD). Compared to the 2D adaptive BF, a 3D BF may have more flexibility in reusing the radio resources in the spatial domain.

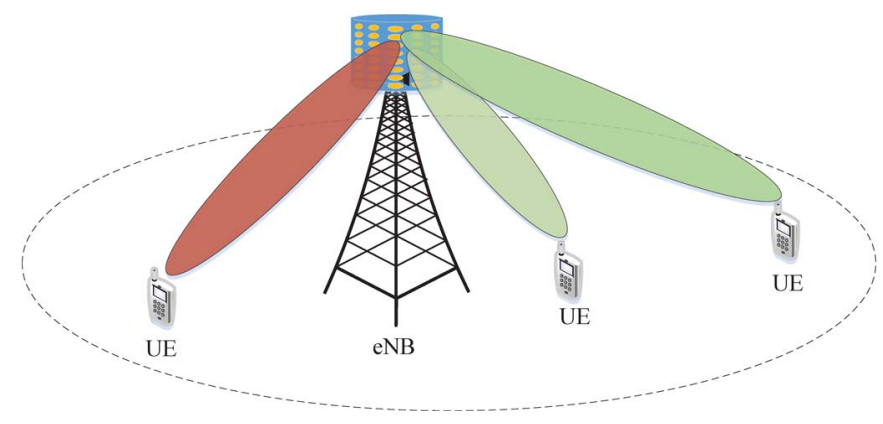

Fig. 5. Illustration of Case 1B: Adaptive beamforming.

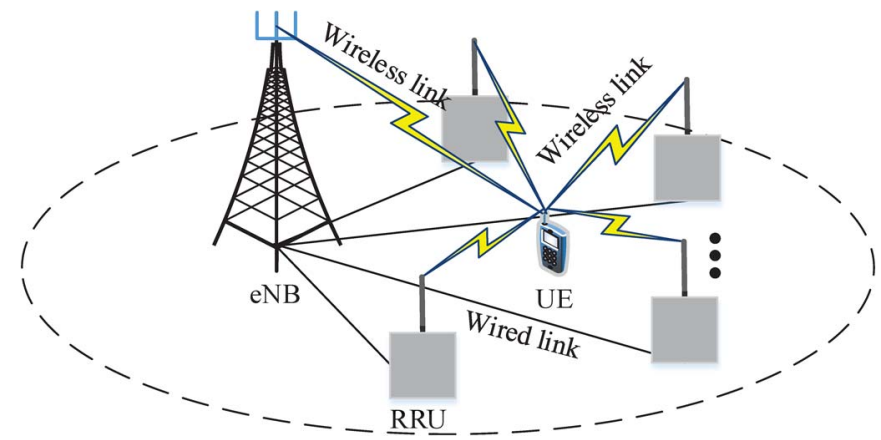

Fig. 6. Illustration of Case 1C: Large-scale cooperation.

3) Case 1C-Large-scale cooperation: Most of the existing contributions on LS-MIMOs show different benefits in a colocated deployment scenario, where there is a large number of antennas installed at a single cell site. However, such co-located deployments impose challenges both on their hardware design and on their field deployment. On the other hand, Distributed Antenna Systems (DASs) associated with spatially separated antennas have been conceived for improving the indoor coverage using a moderate number of antennas [47]. Recent studies have shown that apart from its improved coverage, a DAS is capable of significantly increasing the network's BE, even in the presence of ICI [48]. This motivates researchers to identify specific scenarios as illustrated in Fig. 6, where the LS-MIMO system associated with a distributed architecture outperforms the one relying on a co-located deployment [49], [50]. 


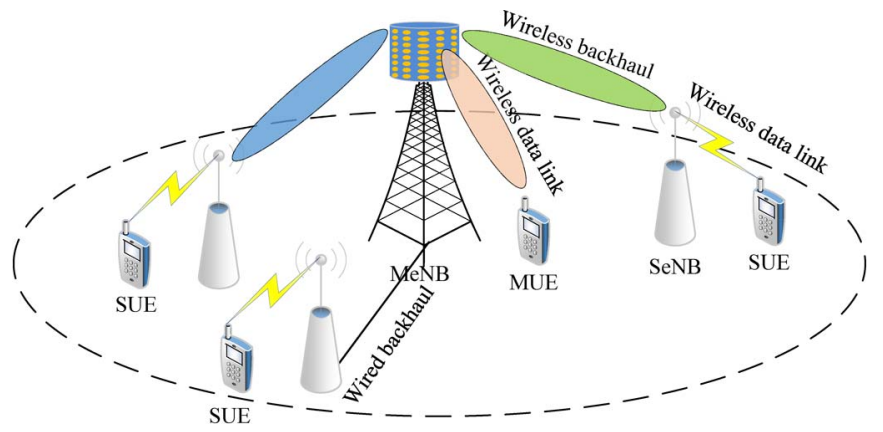

Fig. 7. Illustration of Case 2A: Wireless backhaul.

The advantage of distributed LS-MIMOs is plausible, because the signals arriving from the distributed antennas to each UE are subject to independent random levels of large-scale fading, thereby leading to potential capacity gains over their co-located counterpart [51]. However, it may be a challenge to achieve these gains by coordinating the intra-cell interferences, especially in scenarios having dozens or even hundreds of RRUs in a cell. Although full cooperation constitutes an efficient method of eliminating the intra-cell interference, it is not practical due to its high reliance on full CSI sharing. To strike an elegant trade-off between the performance attained and the overhead imposed, efficient large-scale cooperation schemes are of high importance under this scenario.

Moreover, distributed LS-MIMO and small cell deployments may be viewed as being complementary rather than competitive. For example, a cooperative cellular architecture composed of a DAS and a femtocell-macrocell underlay system is proposed in [51], which may be extended to operate in conjunction with distributed LS-MIMOs.

\section{B. Cases 2: HetNet Scenarios}

1) Case 2A-Wireless Backhaul: The HetNet with dense small cells has been regarded as a very promising design architecture in terms of energy and area BE. It typically consists of multiple types of radio access nodes, e.g., a Macro-cell eNB (MeNB) and multiple Small-cell eNBs (SeNBs) such as pico, femto and relay eNBs. All SeNBs need to be connected to their donor MeNBs through a wired or wireless backhaul. Generally, the wireless backhaul is preferred to instead of the wired backhaul because of easy deployment. In this scenario, an LS-MIMO is used at the MeNB, which has a high DoF so to support multiple wireless backhauls in the HetNet [52].

As illustrated in Fig. 7, the same spectrum may be reused among wireless backhauls, access of Macro-cell UEs (MUEs) and Small-cell UEs (SUEs). In other words, SeNBs can be viewed as a special kind of UEs communicating with the MeNB via the wireless backhaul. Since the location of an eNB is usually fixed, the channel of the wireless backhaul may be quasi-static time varying. Therefore, the MeNB is capable of eliminating the interference between the wireless backhaul and MUEs through the use of precoding.

2) Case 2B-Hotspot Coverage: Statistics show that the majority of tele-traffic originates from buildings, such as super-

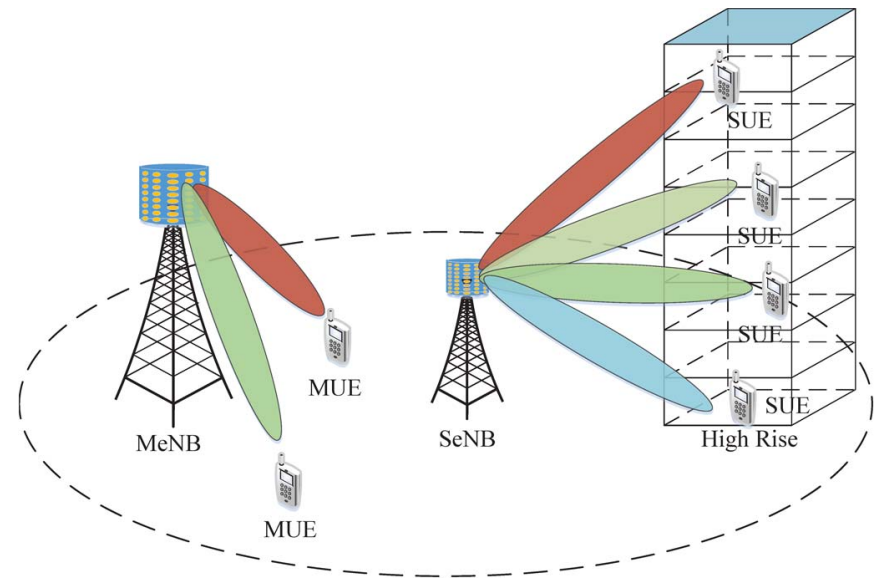

Fig. 8. Illustration of Case 2B: Hotspot coverage.

markets, office buildings, gymnasiums and so on [1]. Therefore, high quality indoor coverage of buildings is considered as one of the key scenarios for the HetNet. Since the tele-traffic is generated at different heights in buildings, traditional AAs with a fixed Downlink (DL) tilt, which are mainly designed for UEs roaming at the street level, are no longer suitable for this scenario. A massive AA is capable of dynamically adjusting both the azimuth and elevation angles of its beam. It can transmit the beams directly to the UEs at different floors in a building, and thus significantly improves system throughput [53]. However, when the indoor coverage of the building is provided by the MeNB with a massive AA, the adjustable range of the elevation angles remains small compared to that of the SeNB, and the angular resolution cannot meet the needs of UEs, as shown in Fig. 8. As it is well known, the close distance between SeNBs and SUEs results in reduced path losses. Therefore, the SeNBs equipped with a massive AA are more appropriate for in-building coverage, providing that deployment costs are acceptable.

3) Case 2C-Dynamic Cell: Since the Reference Signal Received Power (RSRP) gleaned from the MeNB is usually higher than that from the SeNB in HetNets, more UEs are likely to be connected to the MeNB, leading to a potential unbalanced traffic distribution between the macrocell and small cells. The Cell Range Extension (CRE) technique may be used for offloading the traffic from the macro-cells to small cells [54]. However, the UEs in the extended range, which are somehow forced to access to the small cells, may experience low SINRs due to the strong interference encountering from the MeNB. This may cause the unreliable communications between them and SeNBs. In order to solve this problem, the Almost Blank Subframe (ABS) technique can be applied to reduce the interference from the MeNB through time domain coordination [55]. In other words, the QoS performances of SUEs in the extended range is improved at the expense of multiplexing gains.

With the introduction of massive AAs into SeNBs, the down tilt of the transmit signals is adjustable achieve a better received signal quality at SUEs. As illustrated in Fig. 9, it is helpful in adaptively expanding or shrinking the radius of small cells, i.e., Dynamic cell. Therefore, the UEs at the edge of the small cell 


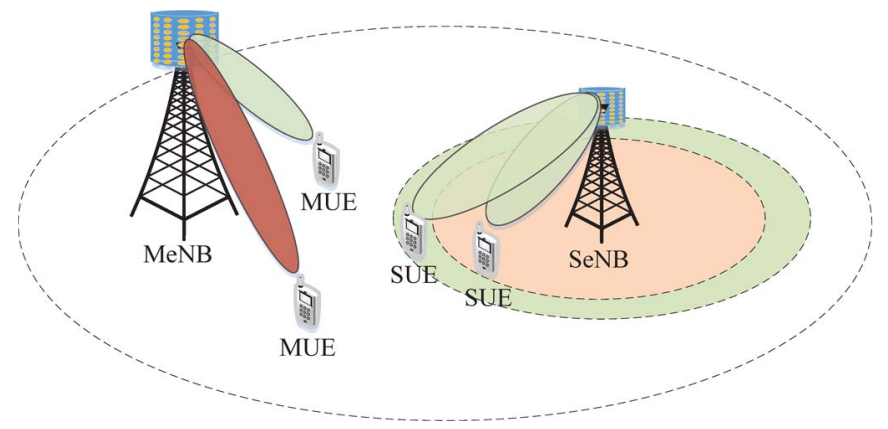

Fig. 9. Illustration on Case 2C: Dynamic cell.

may opt for adaptively connecting to the SeNB according to their received power level. It is appropriate for balancing the traffic between the macro-cell and small cells in the extended range [15], [53].

\section{Conclusion of Application Scenarios}

In this section, typical application scenarios are classified into two types, i.e., homogeneous and heterogeneous networks with LS-MIMO. The former is with only macro-cell deployment, which includes multi-layer sectorization, adaptive beamforming and large-scale cooperation. Multi-layer sectorization is capable of increasing multiplexing gains through splitting the sectors. Adaptive beamforming focuses the radiated energy in the desired direction with the aid of an extremely narrow beam, which is able to improve the desired SINR of the UE, whilst simultaneously reducing the interference imposed on other UEs. Compared to the conventional DAS technique, largescale cooperation, through scaling up the number of distributed antennas that are coordinated, is capable of further enhancing both coverage and achievable throughput.

There are three typical application scenarios in the case of the HomoNet with LS-MIMO. Firstly, the employment of a wireless backhaul by LS-MIMO between MeNB and SeNBs is more flexible and of low cost compared to its wired backhaul counterpart. Then, the SeNB with a massive AA is able to adaptively adjust both the azimuth and elevation angles in an effort to improve the coverage of indoor hotspots, e.g., in buildings. Moreover, the cell radius in HetNet is dynamically adjustable so as balance the load between MeNB and SeNBs by changing the elevation angle.

Based upon the above discussions, LS-MIMO is expected to be applied in numerous scenarios to improve achievable capacity and throughput. However, extensive studies are still needed in practical network deployment.

\section{Physical Layer Technology}

When an eNB equipped with $N$ antennas serves $K(\ll N)$ single-antenna aided UEs in a single-cell environment, the performance bound of LS-MIMO can be readily derived by means of information theory. The attainable multiplexing gain equals the number of receive antennas $K$, and the array gain is proportional to the number of transmit antennas $N$ [12]. However, in a practical system the realistically achievable sum rates depend on the associated coding, modulation and precoding arrangements. As LS-MIMO constitutes an emerging technology, the physical layer techniques conceived for LS-MIMO systems focus primarily on either TPC or on sophisticated detection arrangements [12]. Therefore, the performance of diverse LSMIMO relying on different TPCs or detectors and operating in both single-cell and multi-cell environments is discussed in this section. Both the associated theoretical and measured results indicate that the employment of simple linear TPC and detectors achieves almost the same performance as that of highcomplexity non-linear TPC and detecters, at least in the singlecell scenario. Pilot contamination is the primary performance limiting factor of LS-MIMO, when linear channel estimation algorithms are adopted in the multi-cell environment. Hence, solutions capable of tackling pilot contamination are also discussed. Finally, a range of non-ideal factors degrading the performance of LS-MIMO are addressed.

\section{A. Performance of Precoders/Detectors in Single-Cell Environments}

1) Theoretical Performance: Employing the CBSM, including both the i.i.d. Rayleigh channel model and correlated Rayleigh channel model, the performance of different TPCs or detectors designed for LS-MIMO has been widely analyzed in single-cell environments [12], [17], [56], [57], etc.

(a) i.i.d. Rayleigh channel:

- Linear precoder/detector: When employing a linear detector, the BE performance lower bounds of the Uplink (UL) of LS-MIMO systems have been studied both with perfect CSI and with the aid of realistically estimated CSI [17]. A Maximum Ratio Combining (MRC) receiver performs worse than its Zero-Forcing (ZF) and Minimum Mean Square Error (MMSE) criterion based counterparts in the high Signal-to-Noise Ratio (SNR) region [17]. However, when the SNR is low, the MRC detector outperforms the ZF and MMSE detectors, since the IUI imposed by the MRC detector falls below the noise level [17]. Moreover, the radiated power of the UE can be made inversely proportional to the number of AEs at the eNB under the idealized conditions of having perfect CSI. However, in the presence of realistically estimated CSI, it is only inversely proportional to the square-root of the number of AEs [17]. The relationship between the radiated $\mathrm{EE}$ and $\mathrm{BE}$ has also been investigated. Upon increasing the $\mathrm{BE}$, the $\mathrm{EE}$ of MRC is initially better, but beyond a crossover point it becomes worse than that of ZF [17].

Similar to [17], the BE and radiated EE recorded for the DL of LS-MIMO systems are also evaluated by considering both the realistically estimated CSI and the CSI overhead imposed by the pilots [56]. The lower bounds of $\mathrm{BE}$ and the optimal number of UEs supported are studied for both the Maximum Ratio Transmission (MRT) based TPC as well as for ZF TPC. Based on the lower bound, the radiated $\mathrm{EE}$ as a function of the $\mathrm{BE}$ is quantified [56], which is found to be a monotonically decreasing function of the BE for both detectors. Upon increasing the BE, the EE of MRT is initially better than that of ZF, and then 
the opposite trend holds. In the high-EE regime, the total computational complexity of the MRT TPC may be higher than that of the ZF TPC, since its optimal number of UEs is higher. However, it may still be preferable to $\mathrm{ZF}$, since MRT TPCs can be realized with the aid of a de-centralized architecture [58].

- Non-linear precoder: Apart from the above-mentioned linear TPCs, non-linear TPCs, such as the DPC [59], the Vector Perturbation (VP) [60] and the lattice-aided TPC methods [61] are also investigated and compared to linear precoders for transmission over the i.i.d. Rayleigh channel [12]. The performance of the ZF TPC is shown to approach that of the DPC TPC, when the number of AEs increases. Similarly, diverse non-linear detectors, such as the MMSE based Soft Interference Cancellation (MMSESIC) scheme [62], the Block-Iterative Generalized Decision Feedback Equalizer (BI-GDFE) [63], Tabu Search (TS) [64] and the Maximum Posterior Probability (MAP) algorithm are also evaluated in terms of the Bit Error Ratio (BER) versus complexity [12].

Moreover, the so-called per-antenna Constant Envelope Precoding (CEP) technique implemented with the aid of a sub-optimal algorithm is also invoked for the DL of LSMIMO systems in an attempt to improve the efficiency of their Power Amplifiers (PAs) [65]. It is stated in [65] that an eNB relying on CEP needs about $4 \mathrm{~dB}$ lower transmit power than a conventional technique having a high Peak to Average Power Ratio (PAPR), which is an explicit benefit of using an efficient PA.

(b) Correlated Rayleigh channel: When considering both a realistic Doppler-induced correlation and imperfect CSI, the sum rates of the ZF and Regularized ZF (RZF) [66] TPCs were studied in the DL of an LS-MIMO system in [67]. Since the sum rate of ZF precoding first increases and then decreases with the number of UEs, hence the optimal number of UEs is obtained in [67]. Similarly, the optimal parameters of the RZF TPC may be determined by finding the optimal sum rate. Moreover, the optimal power allocation scheme of both ZF and RZF TPCs, as well as the optimal amount of feedback required in Frequency Division Duplex (FDD)/Time Division Duplex (TDD) systems are also determined in [67] for LS-MIMO systems.

Both the ZF and Correlation Rotation (CR) aided TPC techniques in [68] are evaluated in terms of both the BE and the Symbol Error Ratio (SER), considering the effects of both transmit correlation as well as mutual coupling [57], [68]. According to the approximate lower bounds and to the simulation results characterizing both TPCs, CR-aided precoding outperforms ZF precoding. Upon increasing the number of AEs within a given antenna dimension, the increased transmit diversity achieved dominates the attainable performance, rather than the reduction in spatial diversity imposed by shrinking the spacing among the antennas.

2) Measurement Performances: All the above-mentioned results are based on the use of theoretical channel models, such as the i.i.d. Rayleigh and correlated channel models. However, there exist some differences between the theoretical channel models and their practical counterparts. Therefore, the performances of different TPCs are also measured in practice.

- Effects of correlation [29], [30]: Both the theoretical and realistic sum-rates versus the SNRs of the DPC, RZF and MRT precoders are measured, when the eNB employs a 112-element AA in an outdoor scenario [30]. The theoretical performance bound can be derived, despite significant differences between the i.i.d. Rayleigh channel model and the realistic channel. The correlation amongst the CIRs of the different UEs decreases upon increasing the number of AEs employed at the eNB, because larger AAs are capable of resolving smaller AoA and CIR differences amongst their channels. Furthermore, when encountering different channel correlations amongst the UE channels, the measured and theoretical sum-rates of the DPC, ZF and RZF TPCs are compared in conjunction with an eNB equipped with a 128-antenna indoor cylindrical array operated in residential areas [29]. Upon increasing the number of antennas at the eNB, the channel correlation decreases, and the measured sum-rates approach their theoretical limits. When the eNB employs 20 antennas, about $98 \%$ of the sum-rate of the ideal DPC scheme is achieved for a pair of single-antenna aided UEs by the ZF or RZF TPCs [29].

- Effects of the propagation environment [28]: Considering realistic environments, both a 128-antenna cylindrical and a linear array are employed at the eNB [28]. Then, their realistic sum-rates are compared for both DPC and ZF TPC to the theoretical sum-rates in the i.i.d. Rayleigh channel [28]. Even for the worst combination of the cylindrical array and a dense population supported in a Line of Sight (LoS) environment, ZF precoding is capable of achieving about 55\% of the DPC scheme's sum-rate in the i.i.d. Rayleigh channel, when the number of antennas exceeded 40. By contrast, in a Non Line of Sight (NLoS) environment, despite encountering a dense user population, both cylindrical and linear arrays relying on ZF TPC are capable of attaining about $80-90 \%$ of the sum-rate of the DPC scheme. Regardless of the propagation environment, most of the theoretical sum-rate of LS-MIMO is achievable by linear precoding, if the eNB employs a sufficiently high number of antennas [28].

- Real time operation [58]: To implement real-time MRT based TPC, de-centralized MRT precoding weights can be locally calculated at each antenna [58]. A prototype using a 64-antenna rectangular array is employed in [58] for simultaneously serving 15 UEs. The sum-rate of decentralized MRT precoding is similar to that of traditional MRT precoding. Adopting 64 antennas was shown to achieve 6.7 -fold sum-rate gains, while consuming only $1 / 64$ of the transmit power compared to a single antenna [58].

3) Simulation Results: The quantitative results of several linear precoders are illustrated in Fig. 10. Unless otherwise specified, the default simulation configurations in this survey are as follows: a) The number of transmit antennas is 128 ; 


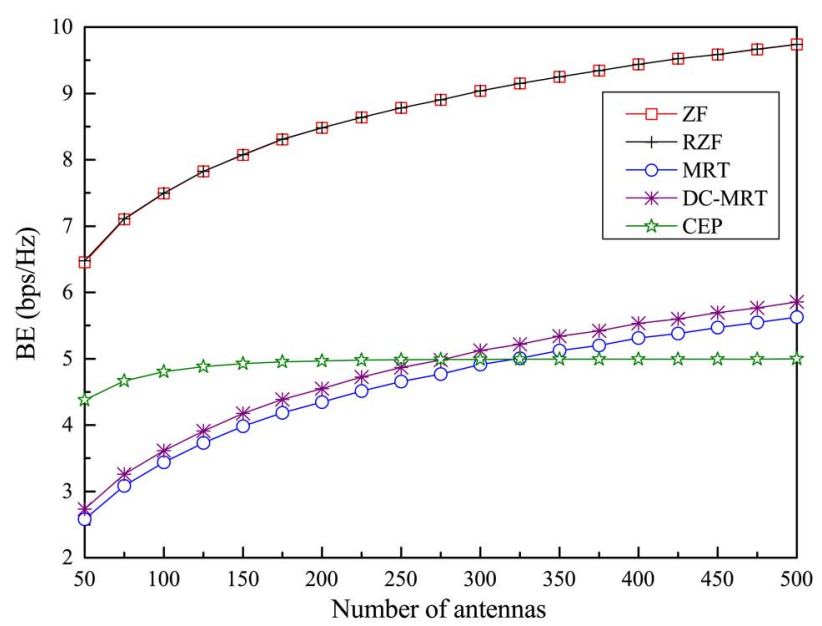

(a)

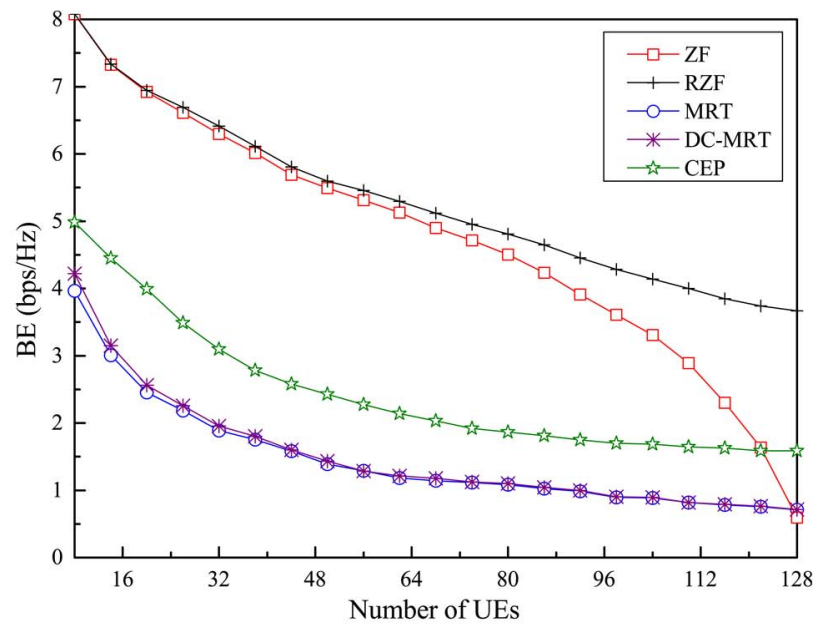

(b)

Fig. 10. BE of a single UE with perfect CSI in single cell case. (a) BE versus the number of antennas. (b) BE versus the number of UEs.

TABLE IV

Precoding and Detection Performance of LS-MiMo in Single-Cell

\begin{tabular}{|r|r|r|r|r|}
\hline & Abbreviation & Full name & Complexity & Metrics \\
\hline \multirow{5}{*}{ Precoder } & $-[58]$ & De-centralized MRT & Low & BE \\
\cline { 2 - 5 } & MRT [30] [56] [58] & Maximum ratio transmission & Low & BE, EE \\
\cline { 2 - 5 } & ZF [29] [28] [56] [67] & Zero forcing & Medium & BE, EE, SER \\
\cline { 2 - 5 } & RZF [29] [30] [67] & Regularized zero forcing & Medium & BE \\
\cline { 2 - 5 } & CR [57], [68] & Correlation rotation precoding & Medium & BE, SER \\
\cline { 2 - 5 } & DPC [12] [28] [29] [30] & Dirty paper coding & High & BE, PAPR \\
\hline \multirow{3}{*}{ Detector } & MRC [17] & Maximum ratio combination & Low & BE, EE \\
\cline { 2 - 5 } & ZF [17] & Zero forcing & Medium & BE, EE \\
\cline { 2 - 5 } & MMSE [17] & Minimum mean-square error & Medium & BE, EE \\
\hline
\end{tabular}

b) Equal power allocation is applied to 10 UEs; c) The system bandwidth is $20 \mathrm{MHz}$; d) The large-scale fading of signal propagation is modeled as $P L[\mathrm{~dB}]=128+37 \log _{10} d$, where $d$ is the distance between the eNB and the given UE in kilometers, the log-normal shadow fading is also considered with the standard variance of $8 \mathrm{~dB}$, and the i.i.d. Rayleigh channel is assumed for the small-scale fading; e) The transmit power at the eNB is $14 \mathrm{dBm}$ and the noise power spectral density is $-174 \mathrm{dBm} / \mathrm{Hz}$; f) In the single-cell scenario, apart from the inner circle associated with a radius of 10 meter, the UEs are uniformly distributed in hexagonal cells, whose ISD is $\mathbf{5 0 0}$ meter.

As there is no performance difference between the CR precoder and the ZF precoder for transmission over the i.i.d. Rayleigh channel, only one of them has to be discussed, say the ZF precoder. As shown in Fig. 10(a), the BE per UE is improved upon increasing the number of antennas at the eNB. However, the gains of the different precoders are not the same for different numbers of antennas. On the other hand, when multiplexing a large number of UEs in the system equipped with a given number of antennas, say 128, the average array gain of each UE is reduced, which impairs the BE in Fig. 10(b). However, the total BE of all UEs is increased as a benefit of the multiplexing gains.
The properties of a variety of LS-MIMO precoders and detectors operating in single-cell environments are summarized in Table IV. Generally, the precoders or detectors associated with a higher complexity offer an improved BE performance. Deploying more number of antennas at the eNB is capable of improving both the BE and EE. The performance of linear precoders or detectors is capable of approximating those of the high-complexity non-linear precoders or detectors. Precoders operating in realistic channels are capable of achieving a BE close to that estimated for idealized theoretical channel models. Assuming ideal CSI or estimated CSI, the transmit power of each antenna can be made inversely proportional to the number of AEs or to the square-root of the number of AEs deployed at the eNB, respectively.

\section{B. Performance of Precoders/Detectors in Multi-Cell Environments}

1) Pilot Contamination in Multi-Cell Scenarios [10], [16], [69], [70]: This section discusses the performance of LS-MIMO in non-cooperative multi-cell multi-user systems. Fig. 11 illustrates the UL and DL interference encountered in such a multi-cell system. The affordable number of orthogonal pilots 


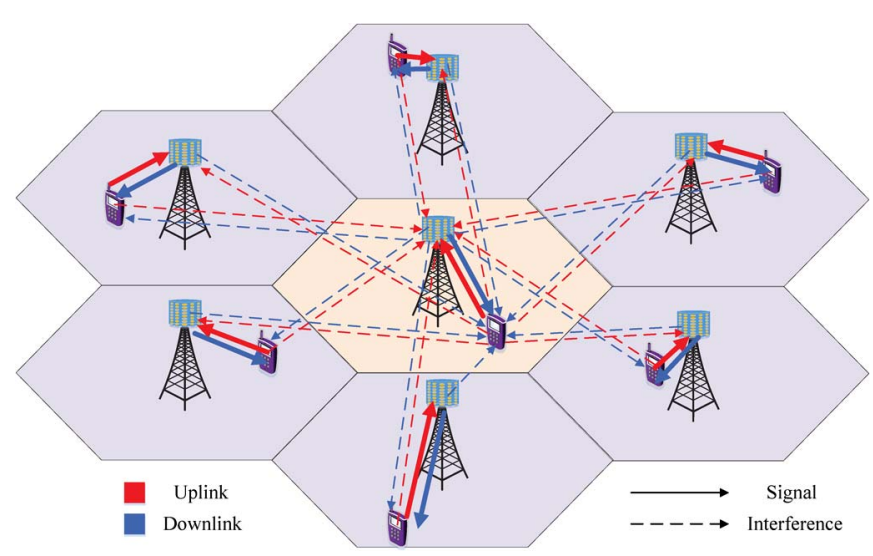

Fig. 11. Illustration of pilot contamination.

used for channel estimation is limited, because increasing the number and variety of pilots is only possible through enlarging the length of the pilot sequences, which ultimately increases the transmission overhead and/or the bandwidth. In practice the number of pilots may only be sufficient for supporting a limited number of UEs roaming in a single cell, which results in inevitable pilot reuse for the UEs in adjacent cells. As shown in Fig. 11, employing the TDD mode, when the UEs of different cells send the same pilot sequence to their donor eNBs on the UL, each eNB has to rely on an interference-contaminated UL received signal, which inevitably contaminates the resultant channel estimate. Firstly, the interference-contaminated UL CSI is used for detecting the UL data. On the other hand, the DL channels may be assumed to be identical to the UL ones in the case of TDD-based reciprocity, provided that the bandwidth remains sufficiently narrow to encounter the non-frequencyselective propagation. Therefore, the UL CIR can be used by the DL transmit precoder. The precoded DL signals impinge on both the desired UEs and the UEs in other cells. Therefore, both the UL and DL sum rates are constrained by the adjacent cells interference imposed by pilot reuse. This phenomenon is known as pilot contamination and has been widely recognized as the main performance constraint of LS-MIMOs, as outlined below in more detail.

- i.i.d. Rayleigh channel: Assuming that the i.i.d. Rayleigh channel can be ideally estimated at the eNB, the performance of the ZF detector is analyzed in a UL LS-MIMO system in the multi-cell scenario [69]. Both the exact closed-form and lower bound of the UL BE indicate that the system is interference-limited at high SNRs. Hence, boosting the transmit power at the UEs cannot further improve the attainable performance. The effects of interference and noise can be reduced by employing more antennas at the eNB. However, in the presence of realistically estimated CSI, the received SINRs of both the MRT precoder and of the MRC detector demonstrate that the effects of the Additive White Gaussian Noise (AWGN) and IUI disappear, and that the only remaining constraint is imposed by pilot contamination [10]. Moreover, the results in [10], [69] demonstrate that the required radiated energy per bit is reduced upon increasing the number of AEs.
- Correlated Rayleigh channel: Under the correlated channel model, the approximate sum rate is studied in [16], where the MRT/RZF precoder is employed for the DL or MRC/MMSE detection is adopted for the UL [16]. The BE attained depends mainly on the effective SNR as well as the DoF, which is defined as the ratio of the rank of the correlation matrix to the number of UEs. Similar conclusions in [16] can be found in [10], i.e., when the number of antennas tends to infinity, both the thermal noise and the IUI can be averaged out. Hence, pilot contamination remains the main constraint of LS-MIMOs, and the radiated power can be kept low. Moreover, the number of antennas required for different TPCs or detectors has to be deduced on the basis of aiming for a given percentage of the $\mathrm{BE}$ attained with the aid of an infinite number of antennas. The RZF/MMSE precoder/reciever is capable of achieving the same $\mathrm{BE}$ using a reduced number of antennas compared to the MRT/MRC schemes.

- Dispersive multi-path channel: The performances of both the MRC and ZF detectors are studied in the UL of a LS-MIMO system in a multi-cell environment, where a dispersive multi-path channel model is assumed [70]. The lower performance bounds derived in [70] demonstrate that pilot contamination remains the dominant performance limiting factor for the LS-MIMO with a realistic finite-dimensional channel model. The ZF receiver performs better than the MRC receiver in terms of the achievable sum-rate, when pilot contamination is not severe, and vice versa. A rich scattering propagation environment may benefit the ZF detector. However, the MRC detector performs better than $\mathrm{ZF}$ in poor scattering propagation environments. If the AoAs of the UEs roaming in the specific cell that uses the same pilots are not identical, pilot contamination can be completely eliminated with the aid of Bayesian channel estimation [33]. In other words, if the covariances of the desired signal and interference span different subspaces, pilot contamination can be eliminated, provided that there are an unlimited number of antennas.

In order to explicitly show the effects of pilot contamination imposed on the different precoders in the multi-cell scenario, the BE per UE under the i.i.d. Rayleigh channel is depicted in Fig. 12. The default simulation configurations are the same as those of the single-cell scenario, except that the number of cells is 7. It can be seen that the performance trends of all precoders involved in the multi-cell scenario are similar to those in the single-cell scenario. However, the BE performances are degraded due to the pilot contamination compared to those seen in Fig. 10. Moreover, the CEP is more sensitive to pilot contamination in contrast to the other precoders.

2) Remedies of Pilot Contamination: As discussed before, when realistic imperfect CSI is acquired at the eNB, the pilot contamination caused by pilot reuse in multi-cell scenarios imposes the ultimate limitation on the attainable performance of LS-MIMO. This subsection studies the main techniques of eliminating or at least alleviating pilot contamination. As 


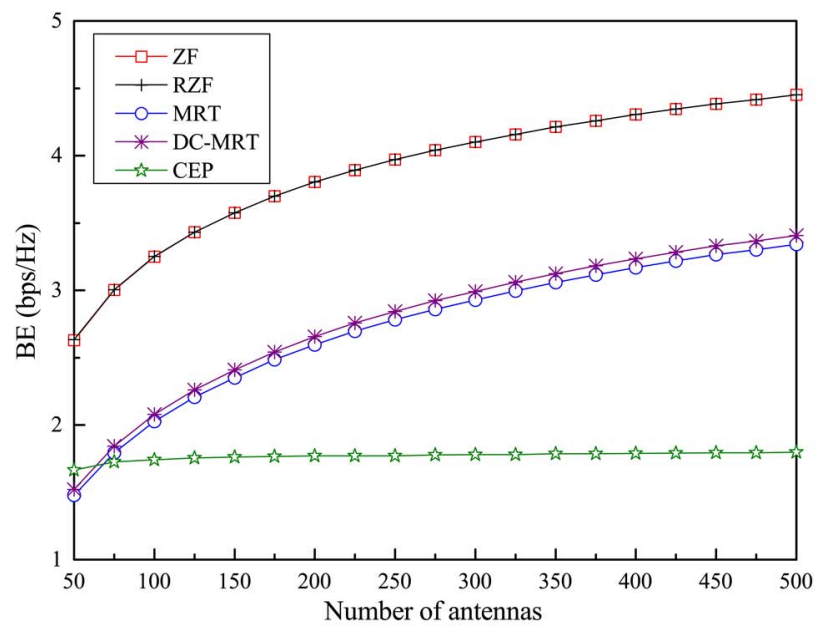

(a)

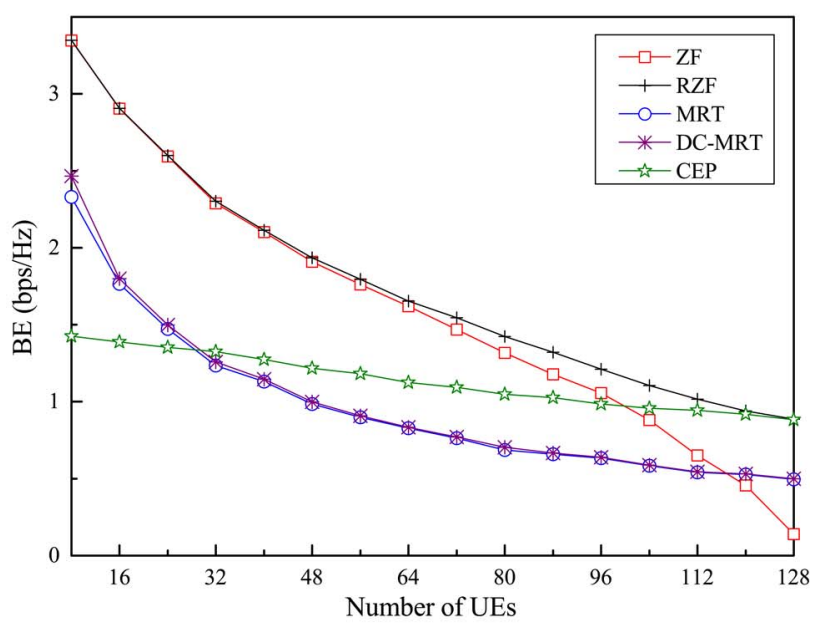

(b)

Fig. 12. BE of single UE for transmission over an i.i.d. Rayleigh channel in multi-cell scenario. (a) BE versus the number of antennas. (b) BE versus the number of UEs.

TABLE V

SCHEMES For Alleviating OR Eliminating Pilot Contamination

\begin{tabular}{|l|r|r|r|r|}
\hline Scheme & Channel model & Shared CSI & Shared Data & Performance \\
\hline Pilot design [71] & i.i.d. Rayleigh & non & non & mitigation \\
\hline \multirow{2}{*}{ Pilot allocation [72] [73] } & i.i.d. Rayleigh & overall large-scale fading & non & mitigation \\
\cline { 2 - 5 } & multi-path & overall covariances & non & rely on PDF of AoAs \\
\hline $\begin{array}{l}\text { Time-shifted pilot [74] } \\
\text { Power allocation [75] }\end{array}$ & Rice & non & non & mitigation \\
\cline { 3 - 5 } Channel estimations [76] [77] & i.i.d. Rayleigh & local large-scale fading & non & overall \\
\hline \multirow{2}{*}{ Precoding [78] [79] } & i.i.d. Rayleigh & overall large-scale fading & overallimination \\
\cline { 2 - 5 } & i.i.d. Rayleigh & estimated aimed and interference channel & non & mitigation \\
\hline
\end{tabular}

concluded in Table V, the main counter-measures can be classified as follows.

- Pilot design: When the classic Matched Filter (MF) is invoked for estimating the UL channel through the use of UL pilots in the i.i.d. Rayleigh channel, a specific pilot design criterion, aiming to minimize the inner product of the pilots for different cells is proposed in [71] for mitigating pilot contamination. Based on this criterion, Chu-sequence-based pilots are designed, which makes the pilots for the UEs in the same cell orthogonal, and reuses the same pilots in the neighboring cells after suboptimal phase shifts. Therefore, the accuracy of the estimated channels can be substantially improved and pilot contamination can be beneficially mitigated.

- Pilot allocation: When the eNB employs an MMSE channel estimator in the i.i.d. Rayleigh channel, pilot contamination is proved to also be the dominant constraint of BE even with the assistance of multi-cell MMSE TPC [72]. However, if we allow the UEs benefitting from low ICI to reuse the same pilots, pilot contamination may be mitigated and substantial BE improvements can be achieved [72].

In [33], [73], a Bayesian channel estimator is first developed for the UL channel in the multi-cell scenario.

Group 1 \|\|$^{\text {Pilot }}\left\|^{\text {Processing }}\right\|^{\text {Downlink }}$ Uplink

Group 2

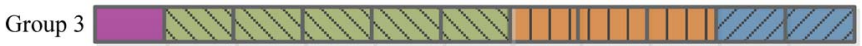

Fig. 13. Time-shifted frame structure with three groups [74].

Then, it is shown that pilot contamination is commensurately reduced, when using more antennas at the eNB, provided that the covariances of the desired signal and interference span different subspaces. Furthermore, a pilot allocation scheme is proposed for suppressing the pilot contamination by carefully shaping the covariance in order to satisfy this condition.

- Frame structure: A time-shifted frame structure is designed in [74] for mitigating pilot contamination based on MF-assisted channel estimation. In this scheme, all the cells are divided into different groups, which transmit their UL pilots in different time slots. When a specific group transmits its UL pilots, the other groups transmit their DL data. An example of the frame structure of three groups is depicted in Fig. 13. The theoretical proof 
TABLE VI

Non-IDEAL FACtors Limiting THE LS-MIMO PERformanCE

\begin{tabular}{|l|r|r|}
\hline Category & Non-ideal factors & Affection \\
\hline \multirow{2}{*}{ Imperfect CSI } & Channel estimation error [16], [78] & Pilot contamination \\
\cline { 2 - 3 } & Channel aging [80] & Mismatched precoding \\
\hline \multirow{3}{*}{ Non-ideal hardware } & Mutual coupling [81] & Energy loss \\
\cline { 2 - 3 } & Pmplifier non-linearities, I/Q imbalance, etc. [83] & Additive distortion noise \\
\cline { 2 - 3 } & Amo ne [82] & Phase drifts \\
\hline
\end{tabular}

provided in [74] shows that the ICI imposed by the different groups is gradually mitigated upon employing more antennas at the eNB. In order to efficiently suppress the ICI arriving from the inner group, optimum power allocation may be employed according to the specific received SINRs on both the UL and DL [74], [75].

- Channel estimation: Taking advantage of the asymptotic orthogonality of different UE channels in the i.i.d. Rayleigh channel, an eigenvalue-decomposition-based approach is proposed in [76] to improve the channel estimation accuracy. Hence, the fast fading channel coefficients may be estimated with the aid of joint estimation of the channels and data by blind techniques, whilst mitigating pilot contamination [11], [77].

- Precoding scheme: A pilot contamination mitigating precoding scheme is designed in [78] for communicating over an i.i.d. Rayleigh channel. This two-stage precoding scheme consists of an outer multi-cell TPC arrangement based on the knowledge of large-scale fading features, and conventional inner linear TPC based on the estimated fast fading coefficients. The essential idea behind this scheme is that the eNBs linearly combine their signals intended for all the UEs by reusing the same pilots. The combined symbols are then transmitted with the aid of traditional precoding. As a result, the ICI can be completely eliminated, as shown by the theoretical derivations in [78].

A further optimization problem is formulated in [79] to mitigate pilot contamination. The objective function used is constituted by the Mean Square Error (MSE) of the received signals of the UEs in the same cell, plus the mean-square interference power imposed on the UEs in other cells. Then, the optimal closed-form expression of precoder for this MMSE-based multi-cell precoding optimization problem is derived analytically. The proposed precoding scheme is capable of simultaneously reducing both the intra-cell and inter-cell interferences. It is implemented at each eNB without information exchange among eNBs, which has the benefit of lower overhead than the joint-multi-cell precoding in [78].

\section{Non-Ideal Factors Limiting the Performance of LS-MIMO}

In a realistic scenario, two main types of non-ideal factors limit the attainable performance of the LS-MIMO system, namely the imperfect CSI and practical hardware implementation, which are listed in Table VI. Naturally, realistic channel estimation is carried out at the DL receiver and its UL signaling delay results in imperfect CSI at the transmitter, whereas non- ideal hardware is attributed mainly to the deleterious effects of mutual coupling, non-linear amplification, I/Q-imbalance and phase noise, which are the widely known imperfections of practical transceivers.

1) Imperfect CSI:

- Imperfect channel estimation: Since in practice both the precoder and the detector have to rely on realistically estimated CSI, accurate channel estimation is vital for the performance of the LS-MIMO system. Under the TDD mode, due to pilot reuse in multi-cell scenarios, the CSI obtained by linear channel estimators, such as the MMSE and MF estimators, is contaminated by interference [16], [78]. In order to eliminate or mitigate the pilot overhead, blind channel estimation is considered in [76]. Under the FDD mode, the feedback from UEs to eNBs may become overwhelming for practical systems, which can be mitigated by compressive sensing [84]. However, channel estimation for LS-MIMO, such as a $100 \times 10$ element system remains a challenging problem, since the complexity of estimating or recovering 1,000 channels is excessive. Therefore, non-coherent MIMO also has to be studied.

- Channel ageing: Adopting an autoregressive model for assessing the predictability of a realistic channel, the effect of channel ageing was investigated for LS-MIMO system in [80]. Channel ageing results in a mismatch between the current channel to be encountered by the next transmission and the channel detected at the DL receiver, quantized and sent back for precoding. In order to mitigate the effects of channel ageing on the sum rate of LSMIMO, an optimal causal linear Finite Impulse Response (FIR) Wiener channel predictor is employed in [80] and as a benefit, its sum rate is substantially improved.

In a practical scenario having an imperfect CSI can be characterized for example by the correlation coefficient between the estimated CSI and the perfect CSI. At the time of writing, there are two main methods of modeling the correlation coefficient, namely the Gaussian model [85] and the Clarke model [86]. They describe the correlation coefficient as the function of Doppler frequency shift and channel delay in the form of either an exponential function or the zeroth-order Bessel function, respectively. When the correlation coefficient calculated by the Gaussian model is 0.9 , the quantitative performances of the different linear precoders are shown in Fig. 14. As expected, the $\mathrm{BE}$ is reduced due to the inaccurate CSI compared to that seen in Fig. 10. Therefore, advanced techniques are required for mitigating the effects of imperfect CSI. 


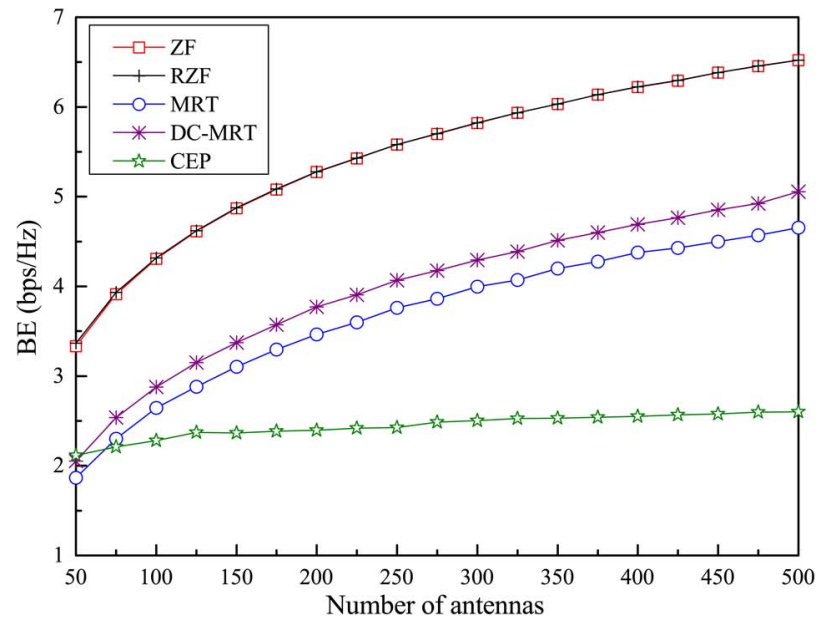

(a)

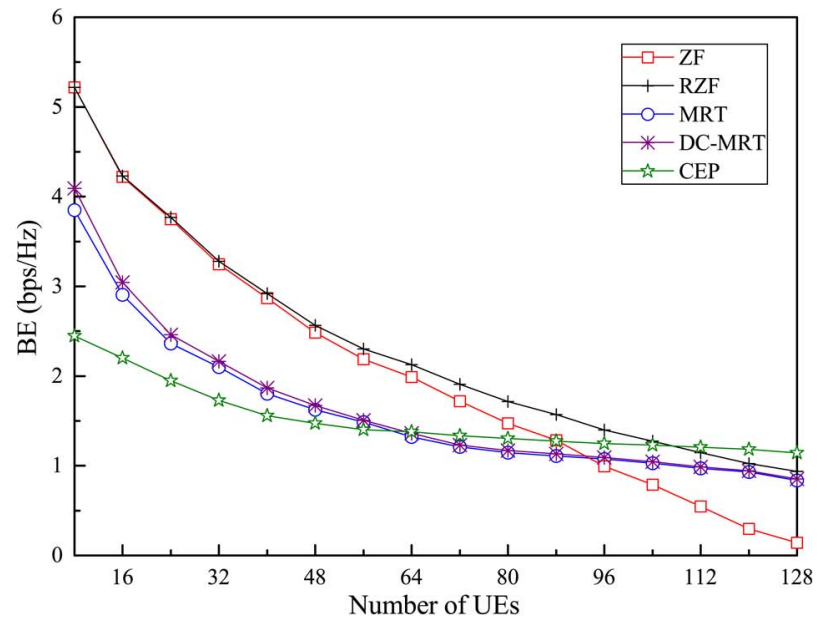

(b)

Fig. 14. BE of single UE with imperfect CSI in single cell scenario. (a) BE versus the number of antennas. (b) BE versus the number of UEs.

\section{2) Non-Ideal Hardware:}

- Mutual coupling: Upon increasing the number of antennas at the eNB, either the spacing between the AEs is reduced, or alternatively, if the spacing is fixed, then the total AA dimension is increased. Having a reduced $\mathrm{AE}$ spacing leads to increased mutual coupling, which impairs the performance of LS-MIMO. In order to mitigate the effects of mutual coupling, sophisticated radiofrequency matching techniques have to be employed between the AA and the radio-frequency chain. In [81], the optimal matching network is designed for circular arrays, which substantially improves attainable system capacity.

- Phase noise: The phase noise is caused mainly by the upconversion stage used at the transmitter and by the downconversion circuit of the receiver. When considering the UL of an LS-MIMO system, the phase noise of the UE transmitter is independent of the phase noise of all other non-cooperative UEs. By contrast, the phase noise of different antennas at the eNB is dependent on whether a single-oscillator centralized eNB or a distinct-oscillator based distributed eNB is considered. When adopting MRC detection, the lower bounds of the sum-rate found for both type of solutions suggest that the achievable array gain is proportional to the square-root of the number of antennas [82]. Due to the progressive phase noise drift at the oscillators, there exists a fundamental trade-off between the time interval used for data transmission and the sum-rate. This trade-off offers an insight into the impact of transmission parameters.

- Additive distortion of the baseband: The amplifier nonlinearity, the I/Q imbalance and phase noise, as well as the additive distortion terms encountered at the transceivers of both the eNB and UEs were introduced into the system model in [83]. Both the channel estimation accuracy and the sum rate of UEs are predominantly limited by the distortion encountered by the UEs, not by the eNBs. Both the impact of the distortion imposed on the eNB and pilot contamination were mitigated by deploying more number of antennas in [83]. Moreover, the attainable EE can be improved by employing more number of transmit antennas, while simultaneously reducing the radiated power. Therefore, the performance degradation imposed by lowcost AEs can be mitigated by increasing the number of transmit antennas.

\section{Summary of Physical Layer Issues}

In this section, we mainly discussed the performance of diverse TPCs and detectors for LS-MIMO systems. In the single-cell scenario, low-complexity linear precoders and detectors may perform similarly to other complex precoders and detectors in terms of their sum-rate [12], [17]. The beneficial combination of TPCs and detectors lead to the achievement of a performance close to their theoretical performance under realistic channel conditions. Both the attainable $\mathrm{BE}$ and $\mathrm{EE}$ can be improved by employing more number of antennas at the eNB. When near-perfect CSI can be acquired through channel estimation with high accuracy, the radiated power can be made inversely proportional to the number of transmit antennas at the eNB [17], but only inversely proportional to the square-root of the number of antennas in the presence of realistically estimated CSI. In the multi-cell scenario, upon employing linear channel estimation with moderate complexity, pilot contamination becomes a major obstacle to improve the $\mathrm{BE}$, which is due to the limited number of available pilots. Hence, diverse counter-measures have been conceived for mitigating pilot contamination [71]-[79], such as specially conceived pilot design [71], power allocation [75] and pilot-decontamination precoding [78]. Finally, the performance of practical LS-MIMO systems is affected by numerous non-ideal factors [78]-[83], including imperfect CSI [78], [80] and non-ideal hardware [81]-[83]. As one of major research issues, the design of non-coherent blind detection or semi-blind assisted LS-MIMO systems has to be explored, which is capable of dispensing much less channel estimation information. 


\section{Networking Technology}

While the underlying physical layer techniques lay the foundation of LS-MIMO systems, networking techniques also play a vital role in practical systems, making them operate more efficiently, reliably and securely. Since the studies on LS-MIMO systems started not too long ago, the research of their upper layers is still in its infancy. Due to their crucial impact on the attainable performance of LS-MIMO systems, networking techniques have gradually attracted considerable interest in both academia and industry.

The effective exploitation of radio resources is one of the main goals to be achieved by networking techniques. Towards this end, several performance indicators are considered, such as the aforementioned BE and EE. Always serving UEs which are experiencing the best channel conditions is surely capable of improving the system's BE. However, this may result in unfair resource allocation, potentially disadvantaging those UEs that suffer from poor channel conditions, such as the UEs located at cell edges. Therefore, apart from BE and EE, the networking techniques usually take into account fairness in order to guarantee a certain level of minimum performance for all UEs. In the remainder of this section, we only focus our attention on two networking techniques, i.e., ICIC and radio resource scheduling, which are two most important issues in wireless networks.

\section{A. Inter-Cell Interference Coordination}

Cellular communication systems suffer from ICI at the cell boundaries, especially when all the channels are fully reused in adjacent cells. As a result, interference mitigation and coordination techniques are needed for alleviating ICI so as to well support frequency reuse. Here, we focus only on static or semi-static ICIC approaches for LS-MIMO systems in different network deployments. Since dynamic ICIC can be regarded as some kind of multi-cell scheduling schemes, it is left to be discussed later.

1) Homogeneous Networks: ICIC techniques, such as Fractional Frequency Reuse (FFR) [87] and Soft Frequency Reuse (SFR) [88], have been widely investigated in the context of efficient radio resource management in multi-cell environments in an attempt to coordinate co-channel interference, resulting in improved cell-edge coverage, cell edge data rates and area BE. A large-scale AA provides additional spatial DoF. Therefore, ICIC for LS-MIMO systems is able to exploit the spatial DoF for mitigating ICI by nulling certain spatial direction to the neighboring cell [89].

In an LS-MIMO system, each eNB is equipped with a huge number of antennas, serving its scheduled UEs with beamforming, while trading off its excess DoF against coordinating the interference to other cells within a cluster. Compared with network MIMO, LS-MIMO is preferred to because of its low costs of deploying an excessive number of antennas at the cell site [90]. Under the assumption of the same number of DoF per UE and same amount of channel estimation overhead, LS-MIMO with spatial interference coordination outperforms network MIMO [91].

The 3D MIMO system, one of LS-MIMO systems, has the capability to dynamically adapt the shape of the vertical beam- forming pattern to the UEs at different locations. In other words, the UEs at the cell center and cell edge are covered by different vertical beamforming patterns with specific downtilt such that the received signal power for each UE is maximized. Then, cell sectorization in the 3D MIMO system can be carried out not only along the horizontal but also the vertical axis, which results in increased system throughput [92]. However, the ICI problem becomes more complicated with much more sectors per cell. Therefore, it is not straightforward whether the overall BE performance as well as the cell edge UE can be improved. The preliminary study in [93] shows that dynamic vertical beam pattern adaptation can provide BE performance gains even with either simplified or suboptimum approaches. Meanwhile, there exists some work on coordinated vertical beamforming with well-known ICIC schemes applied in LTE such as FFR [94]. In the literature, there is a lack of comprehensive studies on this issue to date.

2) Heterogeneous Networks: The HetNet is an attractive means of increasing achievable network capacity and of enhancing the coverage area and/or QoE. In a HetNet, small cells as a tier are capable of providing hotspot capacity enhancements, whereas macro cells as another tier are responsible for large area coverage in support of high mobility UEs. However, the MeNBs and SeNBs may interfere with each other, if they use the same time-frequency resources without careful coordination. Fortunately, when the MeNBs, or even the SeNBs, are equipped with a large-scale AA, the AA can provide an additional spatial DoF for multiplexing the data of several UEs onto the same time-frequency resource. Furthermore, it can concentrate the radiated energy precisely on the intended UEs, thereby reducing both the intra- and inter-tier interference. LSMIMO systems are also capable of supporting cooperation in an implicit way between the different tiers in the HetNet for the sake of improving the overall system performance.

To satisfy ever increasing data rate demands, a two-tier TDDbased HetNet is introduced in [95], where the macro-cell tier served by the MeNBs equipped with a large-scale AA is overlaid with the small cell tier of single-antenna SeNBs. Making use of explicit benefits of channel reciprocity under the TDD mode, the MeNBs estimate the UL interference covariance matrix characterizing the interference from the overlay small cells, which can be used for DL ZF based TPC to reduce the interference to the SUEs. The MeNBs with LS-MIMO can significantly improve the $\mathrm{BE}$ of small cells at the expense of a moderate loss of the macro cell performance. Additionally, the SeNBs can also be equipped with multiple antennas if needed [96].

Recently, the so-called Reversed TDD (RTDD) protocol has been proposed for the HetNet [97]. In the RTDD protocol as shown in Fig. 15, the sequence of the UL and DL transmission periods in one of the tiers is reversed to the other. For example, in Slot 1, while the MeNB transmits the signals to MUEs in the DL, the SeNB received the signals from SUEs in the UL, and vice versa. In the traditional TDD protocol, the MeNBs and SUEs interfere with each other, and so do the SeNBs and MUEs. The channels between the eNBs and UEs potentially fluctuate rapidly thanks to UE mobility. Therefore, less interference samples are available for approximating the time-averaged 


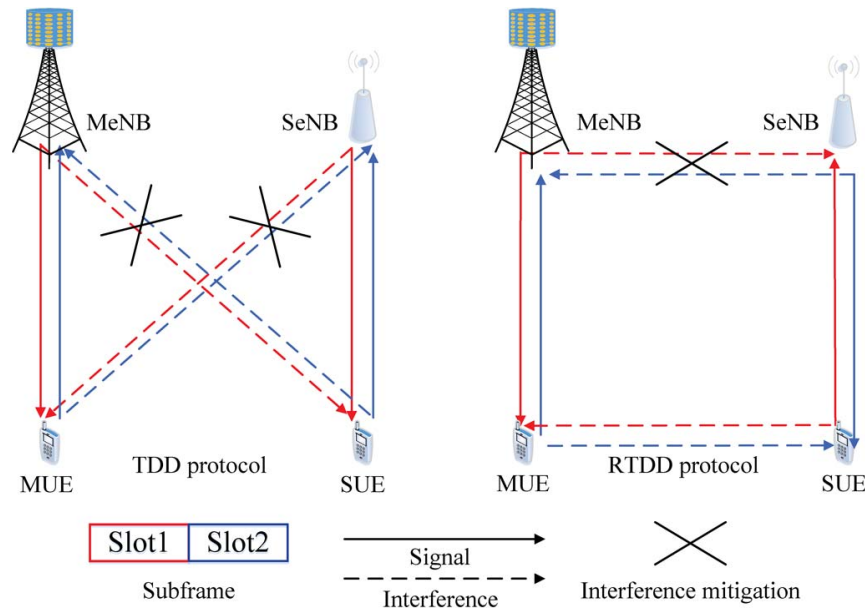

Fig. 15. Illustration of an example with either TDD or RTDD [97].

interference covariance, and hence the resultant estimation errors may degrade the attainable system performance. However, the interference scenario in the HetNet is different if the RTDD protocol is applied, where the MeNB and SeNB interfere with each other, and so do the MUEs and SUEs. Since both the MeNB and SeNB are fixed in location, the interference between them are quasi-static. Hence, the estimated covariance of the channels between them is not sensitive to instantaneous channel variation. Moreover, a massive AA can be deployed at the MeNB and even at the SeNBs so that the interference between the eNBs can be nearly eliminated by narrow beamforming. Meanwhile, the interference between UEs is usually not very serious in most cases because of the low transmission power of UEs. As a result, the RTDD protocol is more suitable for the HetNet with LS-MIMO, since it readily lends itself to cooperative interference cancellation.

\section{B. Scheduling}

Based on the status of queue, channel quality, QoS requirements and so on, the eNB schedules limited radio resources across the time, frequency and spatial domains among the UEs. Different design objectives, e.g., the affordable complexity, overhead, BE, and fairness, are targeted by a variety of scheduling schemes [98]. Usually a good trade-off among all these goals is strived for practical wireless communication systems.

Given a large number of UEs and a limited number of antennas, the problem of sum capacity scaling with UE selection has been widely investigated [99], [100]. In particular, opportunistic beamforming yields significant gains by exploiting the independence of the UEs' channel fluctuation, which may be conducive to achieving multiple UE diversity. Moreover, in order to evaluate the gains of scheduling, the mutual information of the LS-MIMO system may be modeled as a normal distribution under the assumption of i.i.d. Rayleigh fading channels [19]. It turns out that the variance of the channel coefficients grows slowly or even decreases with the number of transmit antennas, but increases with the number of UEs. This conclusion implies that carefully designed robust scheduling schemes would reduce the CSI-feedback rate required.
The more UE-specific and eNB-specific channel information is obtained by the scheduler, the more efficient the system can be. Nonetheless, with increased amount of required information, the overhead and computational complexity for channel information may become prohibitive. Due to the channel hardening phenomenon ${ }^{1}$ of LS-MIMO systems, the scheduling mechanisms relying on full CSI, including large-scale and small-scale prorogation characteristics, are not cost-efficient, because no significant performance gains can be achieved compared to those requiring only partial CSI including the path loss and shadow fading. So, more attention has been paid to scheduling schemes with partial CSI instead of full CSI in LSMIMO systems, both of which are presented for comparison in this section.

1) Full CSI-Based Scheduling: In order to achieve the optimal network performance, the scheduler has to acquire the full and accurate knowledge of all the channels. This knowledge can be exploited to minimize the total power consumption, while satisfying the QoS and power constraints at the eNBs in the HetNet with LS-MIMO [99]. Toward this end, a spatial softcell approach can be taken, where each UE is dynamically assigned to access the optimal nodes, i.e., LS-MIMO MeNB, conventional SeNB or both. If the system assigns a UE to an MeNB and an SeNB at the same time, multiple transmitters serve the UE through joint non-coherent multi-flow beamforming. Also, the total EE can be further improved by applying a low-complexity efficient algorithm, which exploits the hidden convexity in this problem. However, the scheduler has to know the full instantaneous CSI of all the UEs, which incurs both potentially overwhelming estimation overheads and excessive computational complexity due to the large number of AEs and UEs in a practical system. The costs of the scheduling scheme based on full CSI are likely to outweigh the gains.

2) Partial CSI-Based Scheduling: Fortunately, scheduling schemes with partial CSI in LS-MIMO systems are capable of reducing implementation complexity and overhead with an acceptable performance loss.

- Single-cell scheduling: Under the single-cell scenarios, resource allocation for energy-efficient OFDMA systems with a large number of AEs was studied in [101]. Taking into account the associated circuit power dissipation, the imperfect CSI at the Transmitter (CSIT) and QoS requirements, the resources are assigned with the objective of maximizing EE. The considered parameters include the subcarrier allocation, power allocation, antenna allocation and available data rates. It is demonstrated that even though the use of a large number of transmit antennas reduces the multipath-induced fluctuation of each channel, the system performance can still benefit from the different path losses and shadow fading conditions of different UEs. Furthermore, the scheduling scheme can be updated periodically, because the path loss and shadow fading parameters vary slowly, depending on UE mobility.

\footnotetext{
${ }^{1}$ Channel hardening phenomenon is the phenomenon in which the variance of the mutual information (capacity) grows very slowly relative to its mean or even shrinks as the number of antennas grows.
} 
The reduced-complexity probabilistic scheduling algorithms have been proposed in [100], [102], [103], where the number of AEs is quite large. Firstly, different UEs are clustered into groups based on their channel covariance matrices. Then, the UEs in each group are pre-selected randomly based on the group-specific probabilities derived from the whole system. Only the pre-selected UEs are required to transmit their training signals or CSI feedback, leading to markedly reduced overheads and complexity. Moreover, different measures have been conceived both for UE grouping and for scheduling in a FDD based LS-MIMO system based on two-stage precoding, namely on inter-group precoding and intra-group precoding, which are capable of reducing the channel estimation overhead while guaranteeing fairness to the UEs [104].

- Multi-cell scheduling: Unlike the single-cell scenario, both inter-cell and intra-cell interferences have to be taken into account under the multi-cell scenario. Although fully multi-cell scheduling schemes, like the coordinated joint processing in network MIMO, can achieve large performance gains, they are not practical due to prohibitive costs. That is, not only the full CSI but also data streams intended for different mobile users at different cells need to be shared among the eNBs [105]. Instead, another feasible solution is to improve the overall network performance by allowing beamforming vectors from different eNBs to be coordinated for the sake of implementation [106].

The main objective of applying coordinated beamforming in LS-MIMOs is to improve the overall system performance, while reducing the coordination overhead. There are two popular coordinated beamforming schemes, namely the hierarchical [107] and the nested structure [108], [109].

Explicitly, the BF relying on the hierarchical structure [107] at each eNB consists of an inner precoder and an outer precoder. The inner precoder supports the transmission of data to the serving UEs by exploiting the knowledge of the time-variant CSI. Meanwhile, the outer precoder exploits the remaining spatial $\mathrm{DoF}$ for mitigating the ICI by relying only on the knowledge of the average CSI [107]. This structure requires only a modest amount of backhaul overhead and pilots for CSI estimation. Moreover, only the knowledge of the channel's spatial correlation matrices is needed for the BF-weight optimization, which is insensitive to the backhaul latency. By contrast, in the $\mathrm{BF}$ associated with the nested structure, the optimal strategy can be found recursively, where the BF weight optimization Objective Function (OF) may be based on the fairness in power usage subject to satisfying the target SINR constraints [108], [110]. Another nested structure, whose OF focuses on Maximizing the Minimum (MAXMIN) weighted SINR among UEs was proposed in [109], [111]. Unlike the hierarchical structure of [107], the optimal precoder of the nested structure [108], [109] is found as the solution of a joint optimization problem, which aims for striking a trade-off between providing a high SINR for the intra-cell UEs and mitigating the ICI. For instance, when this BF is applied in an LS-
MIMO system, all the eNBs are divided into two groups, 1) Selfish eNBs, whose UE SINRs are relative low; and 2) altruistic eNBs, whose UEs SINRs are relative high. The altruistic group may be empty; or it may use zeroforcing for eliminating the interference by imposing it on the selfish group. If the optimal ZF beamforming scheme is used in the altruistic group, each eNB in this group has to transmit less power than that in the selfish group. The precoder at each eNB uses the optimal BF parameters along with its own instantaneous CSI. Additionally, only the average CSI has to be exchanged among the eNBs.

On the other hand, different metrics can be used to indicate different system performances. Thus, it is crucial to choose an objective with an appropriate metric function for the multi-cell scheduling schemes. There are several kinds of objectives in terms of either efficiency or fairness when multi-cell scheduling is applied in LS-MIMO systems, e.g., a) Minimizing the Maximum (MIN-MAX) the fairness in power consumption subject to certain SINR constraints [108], [110]: It aims to maximize the overall $\mathrm{EE}$ as a high priority by adjusting its coordinated beamforming scheme. A efficient solution can be obtained through Lagrange duality and random matrix theory; b) MAX-MIN SINR subject to certain sum-rate constraints [109], [111]: It enforces the overall system fairness by guaranteeing each UE's promised SINR. In the case of non-convex optimization problems, the optimal scheme may be derived by using nonlinear Perron-Frobenius theory; c) Maximizing the weighted sum rate subject to some eNBs power consumption constraints: This objective can be viewed as a combination of MAX-MIN fairness and maximum sum-rate. Moreover, efficient schemes can be obtained through hidden convexity and random matrix theory [107].

\section{Conclusions on Networking Techniques}

In this section, a wide range of networking techniques conceived for LS-MIMO systems have been investigated, with an emphasis on the associated ICIC and scheduling issues. Since LS-MIMO systems have only been proposed recently, relevant studies are not comprehensive in the literature. As for ICIC, the beamforming for LS-MIMO systems, which helps eliminate the ICI at the expense of computational, and 3D MIMO beamforming, which can be used for cell splitting with low complexity, have been discussed in the context of the HomoNet. The R-TDD protocol, which is helpful for interference cancellation, has also been studied in the context of the HetNet. In scheduling schemes, existing algorithms have been classified according to their requirements in terms of CSI and their different design objectives. The primary objective of scheduling is to improve the attainable system performance, while maintaining affordable implementation complexity and overhead.

\section{Challenges And Open Issues}

The investigation of LS-MIMO has stimulated strong research interest in both academia and industry alike. However, 
much more efforts are needed for practical applications of LS-MIMO in the near future. Based on our discussions in the preceding sections, the following challenges and open issues lie ahead.

\section{A. Design of AAs}

Finding practical antenna configurations is an important issue for LS-MIMO systems, because it affects not only the channel properties, but also their array gains, diversity gains and multiplexing gains. Moreover, AAs may be constrained to a limited physical size. Therefore, the employment of antennas has to be carefully designed to enhance the attainable performance given the physical area constraint.

\section{B. Channel Models}

Channel models have been established for characterizing both 2D and 3D AAs. The elevation angle of 3D AAs has been considered for three different scenarios in 3GPP. However, no channel model has been considered for realistic systems relying on spherical or cylindrical AAs. Hence, more measurements have to be carried out for realistic AAs.

\section{Green LS-MIMO}

With increasing carbon emissions imposed by the excessive power consumption of wireless communications networks, EE has become a significant performance metric of evaluating future wireless networks. However, the evaluation of $\mathrm{EE}$ in LS-MIMO systems in the literature focuses primarily on the EE in the single-cell environment. Furthermore, only the radiated power of the PAs is considered, while ignoring the power dissipation of signal processing circuit and the efficiency of the PAs [17], [56]. Therefore, the realistic EE performance of LS-MIMO systems needs to be analyzed by considering both the radiated power and circuit power in multi-cell scenarios.

\section{Performance Analysis of Large-Scale DASs}

Centralized and distributed antenna deployments constitute two important scenarios of LS-MIMO systems. The majority of current work focuses on the family of centralized LS-MIMO systems. As argued, large-scale DASs are capable of significantly improving both the coverage quality and the capacity of wireless communications networks. The coverage quality of both indoor and outdoor environments should be evaluated in the near-future in terms of the PDF of their SINRs. Stochastic geometry has been widely used for the analysis of wireless networks, with an emphasis on the 2D plane. However, stochastic geometry needs to be extended to the 3D space to analyze largescale DASs.

\section{E. Control Channel of LS-MIMO Systems}

When UEs are powered up, they first receive broadcast information from control channels, which is the first essential step for the UEs to connect to a wireless network. However, the UEs are oblivious of the eNBs during the initial stage, and the power level of the signal transmitted from the eNB is weak compared to that of the PDSCH. This problem occurs not only in the current systems, but also in LS-MIMO systems, since the latter rely on high beam gains. Therefore, effective countermeasures have to tackle this issue in LS-MIMO systems for practical applications.

\section{F. Complexity Versus Performance}

An LS-MIMO system often entails considerably complex signal processing. Hence, substantial efforts have been devoted to streamline and optimize both signal processing algorithms and their implementations. However, low-complexity algorithms generally decrease the performance of LS-MIMO. For example, having more accurate CSI leads to improved performance at the expense of increased processing complexity. When low-complexity linear channel estimation is adopted, the attainable performance is constrained by the pilot contamination phenomenon, especially in multi-cell scenarios. However, pilot contamination can be overcome by using complex channel estimation algorithms [76], [77]. There exists a tradeoff between the complexity of channel prediction as well as the TPC design and the experienced channel, when the UEs move around in a cell. Therefore, simple but efficient algorithms have to be conceived for channel estimation, channel prediction, TPC and detection.

\section{G. Effects of Non-Ideal Hardware}

LS-MIMO relies mainly on the law of large numbers to average out noise, channel fading characteristics, etc. However, in practice, low-cost imperfect components are employed to implement the LS-MIMO system. Therefore, the imperfections of non-ideal hardware, such as the non-linearities of the amplifier, I/Q imbalance, A/D and D/A nonlinearities, should be taken into account [82], [83] in practical LS-MIMO systems. Some existing studies have been reported on evaluating the effects of non-ideal hardware. However, more efforts are required for designing efficient algorithms to mitigate these non-ideal factors.

\section{H. LS-MIMO in the mm-Wave Band}

In the popular frequency bands below $2 \mathrm{GHz}$, the physical size of a large-scale AA is excessive to be feasibly installed in a UE, although it may be readily accommodated by the eNB. In order to avoid this problem, LS-MIMO systems are likely to operate in the millimeter (mm)-wave band [112]. For example, in the $60 \mathrm{GHz}$ band, the corresponding wavelength is only $0.5 \mathrm{~cm}$, hence a large-scale AA having $\lambda / 2$-spaced elements may accommodate as many as 40 AEs within a physical space of $10 \mathrm{~cm}$.

However, the channel measurements indicate propagation losses that are severe in the mm-wave band, hence the propagation distance becomes very short, resulting in small cells [113]. In order to attain both high data rates and a satisfactory coverage, the ultra-dense deployment of small cells operating in the mm-wave band is one of the potential solutions envisaged 
for next-generation wireless communications networks [114]. On the other hand, another challenge is to realize large-scale AAs in the mm-wave band, because it is hard to design practical phased AAs operating at $28 \mathrm{GHz}$. Nonetheless, a large-scale AA prototype with up to 32 miniaturized antennas was reported in [115].

Apart from the relevant physical layer techniques, the network architecture of LS-MIMOs operating in the mm-wave band has to be studied as well. A cellular architecture relying on $\mathrm{mm}$-wave frequencies has been proposed and evaluated, where the MUEs communicating with the MeNB operate in the microwave band and the SUEs use the mm-wave band [116].

Although much attention has been dedicated to the design of LS-MIMO systems in the mm-wave band, there are still many uncertain issues surrounding the practical mm-wave communication systems, such as their hardware design, interference control and resource management.

\section{New Application Scenarios}

Since LS-MIMO is introduced to wireless networks, it has been undergoing rapid changes. As a benefit of its large DoF, the R-TDD scheme has been proposed for alleviating the interference in HetNets. When eNBs are equipped with massive AAs, a wireless backhaul can be established among the eNBs. However, both R-TDD and wireless backhaul techniques are not mature in practice to date, hence studies need to be carried out. Moreover, when a relay node employs a large number of antennas, the channel properties are different from those of a regular relay equipped with a small number of antennas [117].

\section{J. Duplex Mode}

There are two basic duplex modes in wireless communications networks, namely TDD and FDD. Owing to a limited number of orthogonal pilot sequences, TDD is assumed at present in most existing work related to LS-MIMO. A fullduplex system, where the UL and DL transmissions occur simultaneously, is also a good choice. However, preliminary studies on full-duplex systems use only three antennas. Extending the full-duplex research to LS-MIMO systems may be a topic of interest in the near future.

\section{CONCLUSION}

This paper provided a survey on the timely subject of LS-MIMO, including channel modeling, application scenarios, as well as physical layer and networking techniques.

A range of channel measurements and channel models were presented, complemented by a variety of open research issues. Then, a pair of practical application scenarios was discussed, namely HomoNets and HetNets. More specifically, the cases of multi-layer sectorization, adaptive beamforming and largescale cooperation aided HomoNets were studied, followed by the discussion on wireless backhaul design, hotspot coverage and dynamic cell in HeteNets in the specific context of LS-MIMO systems. The design of sophisticated TPCs and detection schemes is vital for achieving the potential gains promised by LS-MIMO systems. Therefore, both their advantages and disadvantages alongside countermeasures were summarized for both the single-cell and realistic multi-cell environments. Moreover, the effects of non-ideal hardware and imperfect CSI have also been discussed, which have to be further studied in the near future.

At the time of this writing, associated networking solutions also attract more attention. The family of coordinated beamforming techniques relying on CSI exchange, low-complexity cell splitting and R-TDD also constitute promising design alternatives for ICIC. The channel hardening phenomenon of LS-MIMO is helpful in terms of reducing the CSI overhead and supporting the development of low-complexity scheduling algorithms, albeit at the cost of limited scheduling gains. In a nutshell, an exciting era for MIMO researchers has come!

\section{REFERENCES}

[1] Global Mobile Data Traffic Forecast Update, 2012-2017, Cisco Visual Networking, May 2013.

[2] Z. Hasan, H. Boostanimehr, and V. K. Bhargava, "Green cellular networks: A survey, some research issues and challenges," IEEE Commun. Surveys Tuts., vol. 13, no. 4, pp. 524-540, 4th Quart. 2011.

[3] D. Feng et al., "A survey of energy-efficient wireless communications," IEEE Commun. Surveys Tuts., vol. 15, no. 1, pp. 167-168, 1st Quart. 2012.

[4] Y. Chen, S. Zhang, S. Xu, and G. Y. Li, "Fundamental trade-offs on green wireless networks," IEEE Commun. Mag., vol. 49, no. 6, pp. 30-37, Jun. 2011.

[5] G. Y. Li et al., "Energy-efficient wireless communications: Tutorial, survey, and open issues," IEEE Wireless Commun., vol. 18, no. 6, pp. 28-35, Dec. 2011.

[6] G. Auer et al., "How much energy is needed to run a wireless network?" IEEE Wireless Commun., vol. 18, no. 5, pp. 40-49, Oct. 2011.

[7] T. Kailath and A. J. Paulraj, "Increasing capacity in wireless broadcast systems using Distributed Transmission/Directional Reception (DTDR)," U.S. Patent 5345 599, Sep. 6, 1994.

[8] G. Golden, C. Foschini, R. Valenzuela, and P. Wolniansky, "Detection algorithm and initial laboratory results using V-BLAST space-time communication architecture," Electron. Lett., vol. 35, no. 1, pp. 14-16, Jan. 1999.

[9] A. Ghrayeb and T. M. Duman, Coding for MIMO Communication System. Hoboken, NJ, USA: Wiley, 2007.

[10] T. Marzetta, "Noncooperative cellular wireless with unlimited numbers of base station antennas," IEEE Trans. Wireless Commun., vol. 9, no. 11, pp. 3590-3600, Nov. 2010.

[11] E. G. Larsson, F. Tufvesson, O. Edfors, and T. L. Marzetta, "Massive MIMO for next generation wireless systems," IEEE Commun. Mag., vol. 52, no. 2, pp. 186-195, Feb. 2014.

[12] F. Rusek et al., "Scaling up MIMO: Opportunities and challenges with very large arrays," IEEE Signal Process. Mag., vol. 30, no. 1, pp. 40-60, Jan. 2013.

[13] Requirements, Candidate Solutions \& Technology Roadmap for LTE R12 Onward, 3GPP RWS-120010, DoCoMo, Jun. 2012.

[14] Technologies for Rel-12 and Onward, 3GPP RWS-120021, Samsung, Nov. 2013.

[15] Views on Rel-12 and Onwards for LTE and UMTS, 3GPP RWS-120006, HUAWEI and HiSilicon, 2013.

[16] J. Hoydis, S. ten Brink, and M. Debbah, "Massive MIMO in the UL/DL of cellular networks: How many antennas do we need?," IEEE J. Sel. Areas Commun., vol. 31, no. 2, pp. 160-171, Feb. 2013.

[17] H. Q. Ngo, E. Larsson, and T. Marzetta, "Energy and spectral efficiency of very large multiuser MIMO systems," IEEE Trans. Commun., vol. 61, no. 4, pp. 1436-1449, Jul. 2013.

[18] L. Zhao, K. Zheng, H. Long, and H. Zhao, "Performance analysis for downlink massive MIMO system with ZF precoding," Trans. Emerging Telecommun. Technol., vol. 25, no. 12, pp. 1219-1230, Dec. 2014.

[19] B. Hochwald, T. Marzetta, and V. Tarokh, "Multiple-antenna channel hardening and its implications for rate feedback and scheduling," IEEE Trans. Inf. Theory, vol. 50, no. 9, pp. 1893-1909, Sep. 2004.

[20] C.-L. I et al., "Toward green and soft: A 5G perspective," IEEE Commun. Mag., vol. 52, no. 2, pp. 66-73, Feb. 2014. 
[21] L. Lu, G. Li, A. Swindlehurst, A. Ashikhmin, and R. Zhang, "An overview of massive MIMO: Benefits and challenges," IEEE J. Sel. Topics Signal Process., vol. 8, no. 5, pp. 742-758, Oct. 2014.

[22] C-RAN: The Road Towards Green Radio Access Network, China Mobile Research Institute, Beijing, China, Oct. 2012.

[23] Y. Wu, R. Zhou, and W. Zhang, "Active antenna system: Utilizing the full potential of radio sources in the spatial domain," Huawei, Shenzhen, China, 2012.

[24] A. Khan and A. Brown, "Null steering in irregularly spaced sparse antenna arrays using aperture distributed subarrays and hybrid optimiser," IET Microw., Antennas Propag., vol. 8, no. 2, pp. 86-92, Jan. 2014.

[25] D. S. Weile, "Orthogonal methods for array synthesis: Theory and the ORAMA computer tool," IEEE Antennas Propag. Mag., vol. 49, no. 6, pp. 131-132, Dec. 2007

[26] A. Antonis, A. G. Kanatas, and C. B. Constantinos, Parasitic Antenna Arrays for Wireless MIMO Systems. New York, NY, USA: SpringerVerlag, 2014.

[27] S. Payami and F. Tufvesson, "Channel measurements and analysis for very large array systems at $2.6 \mathrm{GHz}$," in Proc. 6th EUCAP, Prague, Czech Republic, Mar. 2012, pp. 433-437.

[28] X. Gao, F. Tufvesson, O. Edfors, and F. Rusek, "Measured propagation characteristics for very-large MIMO at $2.6 \mathrm{GHz}$," in Proc. 46th ASILOMAR Conf. Signals, Syst. Comput., Pacific Grove, CA, USA, Nov. 2012, pp. 295-299.

[29] X. Gao, O. Edfors, F. Rusek, and F. Tufvesson, "Linear pre-coding performance in measured very-large MIMO channels," in Proc. IEEE 74th VTC-Fall, San Francisco, CA, USA, Sep. 2011, pp. 1-5.

[30] J. Hoydis, C. Hoek, T. Wild, and S. ten Brink, "Channel measurements for large antenna arrays," in Proc. ISWCS, Paris, France, Aug. 2012, pp. $811-815$

[31] C.-X. Wang, X. Hong, H. Wu, and W. Xu, "Spatial-temporal correlation properties of the 3GPP spatial channel model and the kronecker MIMO channel model," EURASIP J. Wireless Commu. Netw., vol. 2007, no. 1, Feb. 2007, Art. ID. 39871

[32] L. Schumacher, L. T. Berger, and J. Ramiro-Moreno, "Recent advances in propagation characterisation and multiple antenna processing in the 3GPP framework," in Proc. XXVIth URSI Gen. Assem., Prague, Czech Republic, Aug. 2002, pp. 1-9.

[33] H. Yin, D. Gesbert, M. Filippou, and Y. Liu, "A coordinated approach to channel estimation in large-scale multiple-antenna systems," IEEE J. Sel. Areas Commun., vol. 31, no. 2, pp. 264-273, Feb. 2013.

[34] A. Abdi and M. Kaveh, "A space-time correlation model for multielement antenna systems in mobile fading channels," IEEE J. Sel. Areas Commun., vol. 20, no. 3, pp. 550-560, Aug. 2002.

[35] G. Byers and F. Takawira, "Spatially and temporally correlated MIMO channels: Modeling and capacity analysis," IEEE Trans. Veh. Technol., vol. 53, no. 3, pp. 634-643, May 2004.

[36] M. Lu, T. Lo, and J. Litva, "A physical spatio-temporal model of multipath propagation channels," in Proc. IEEE 47th Veh. Technol. Conf., Phoenix, AZ, USA, May 1997, pp. 810-814.

[37] X. Gao, F. Tufvesson, O. Edfors, and F. Rusek, "Channel behavior for very large MIMO systems-Initial characterization," presented at the Proc. Joint Workshop Small Cell Cooperative Communications, Bristol, U.K., Sep. 2012.

[38] L. Liu et al., "The COST 2100 MIMO channel model," IEEE Wireless Commun., vol. 19, no. 6, pp. 92-99, Dec. 2012.

[39] S. Wu et al., "A non-stationary wideband channel model for massive MIMO communication systems," IEEE Trans. Wireless Commun., vol. 14, no. 3, pp. 1434-1446, Mar. 2015.

[40] A. Ghazal et al., "A non-stationary MIMO channel model for highspeed train communication systems," in Proc. IEEE 75th VTC-Fall, Yokohama, Japan, Apr. 2012, pp. 1-5.

[41] J. Meinila et al., "D5.3: WINNER+ final channel models," in CELTIC CP5-026 WINNER, CELTIC, Nov. 2010, pp. 1-107.

[42] Z. Zhong, X. Yin, X. Li, and X. Li, "Extension of ITU IMT-Advanced channel models for elevation domains and line-of-sight scenarios," in Proc. IEEE 78th VTC-Fall, Las Vegas, NV, USA, Sep. 2013, pp. 1-5.

[43] Y.-H. Nam et al., "Full-dimension MIMO (FD-MIMO) for next generation cellular technology," IEEE Commun. Mag., vol. 51, no. 6, pp. 172-179, Jun. 2013.

[44] Technical Specification Group Radio Access Network Study on 3D Channel Model for LTE (Release 12), 3GPP TR-36.873, Oct. 2013.

[45] M. Steinbauer, A. F. Molisch, and E. Bonek, "The double-directional radio channel," IEEE Antennas Propag. Mag., vol. 43, no. 4, pp. 51-63, Aug. 2001.

[46] M. Stege, J. Jelitto, M. Bronzel, and G. Fettweis, "A multiple input-multiple output channel model for simulation of $\mathrm{Tx}^{\mathrm{x}}$ and
Rx-diversity wireless systems," in Proc. 52nd IEEE VTS-Fall, Boston, MA, USA, Sep. 2000, pp. 833-839.

[47] A. Saleh, A. Rustako, and R. Roman, "Distributed antennas for indoor radio communications," IEEE Trans. Commun., vol. 35, no. 12, pp. $1245-1251$, Dec. 1987

[48] R. Heath, T. Wu, Y. H. Kwon, and A. Soong, "Multiuser MIMO in distributed antenna systems with out-of-cell interference," IEEE Trans. Signal Process., vol. 59, no. 10, pp. 4885-4899, Oct. 2011

[49] D. Qiao, Y. Wu, and Y. Chen, "Massive MIMO architecture for 5G networks: Co-located, or distributed?," in Proc. 11th ISWCS, Barcelona, Spain, Aug. 2014, pp. 192-197.

[50] K. Truong and R. Heath, "The viability of distributed antennas for massive MIMO systems," in Proc. 47th ASILOMAR Conf. Signals, Syst. and Comput., Pacific Grove, CA, USA, Nov. 2013, pp. 1318-1323.

[51] Z. Liu and L. Dai, "A comparative study of downlink MIMO cellular networks with co-located and distributed base-station antennas," IEEE Trans. Wireless Commun., vol. 13, no. 11, pp. 6259-6274, Nov. 2014

[52] M. Thomas and H. Yang, "Dedicated LSAS for metro-cell wireless backhaul-Part I: Downlink," Bell Lab., Alcatel-Lucent, Murray Hill, NJ, USA, Tech. Rep., Dec. 2012.

[53] Performance Evaluation of Elevation Beamforming in the Use Scenarios, 3GPP R1-131165, Huawei and HiSilicon, Apr. 2013

[54] Performance Evaluation of Cell Range Extension, 3GPP R1-112894, Huawei and HiSilicon, Jun. 2011.

[55] A. Damnjanovic et al., "A survey on 3GPP heterogeneous networks," IEEE Trans. Wireless Commun., vol. 18, no. 3, pp. 10-21, Jun. 2011.

[56] H. Yang and T. Marzetta, "Performance of conjugate and zero-forcing beamforming in large-scale antenna systems," IEEE J. Sel. Areas Commun., vol. 31, no. 2, pp. 172-179, Feb. 2013.

[57] C. Masouros, M. Sellathurai, and T. Ratnarajah, "Large-scale MIMO transmitters in fixed physical spaces: The effect of transmit correlation and mutual coupling," IEEE Trans. Commun., vol. 61, no. 7, pp. 2794-2804, Jul. 2013.

[58] C. Shepard et al., "Argos: Practical many-antenna base stations," in Proc. ACM Int. Conf. Mobile Comput. Netw., Istanbul, Turkey, Aug. 2012, pp. 53-64.

[59] C. B. Peel, "On dirty-paper coding," IEEE Signal Process. Mag., vol. 20 no. 3, pp. 112-113, Sep. 2003.

[60] B. Hochwald, C. Peel, and A. Swindlehurst, "A vector-perturbation technique for near-capacity multiantenna multiuser communication-Part II: Perturbation," IEEE Trans. Commun., vol. 53, no. 3, pp. 537-544, Mar. 2005.

[61] C. Windpassinger, R. F. H. Fischer, and J. Huber, "Lattice-reductionaided broadcast precoding," IEEE Trans. Commun., vol. 52, no. 12 , pp. 2057-2060, Dec. 2004

[62] Y.-C. Liang, E. Y. Cheu, L. Bai, and G. Pan, "On the relationship between MMSE-SIC and BI-GDFE receivers for large multiple-input multiple-output channels," IEEE Trans. Signal Process., vol. 56, no. 8 , pp. 3627-3637, Aug. 2008

[63] Y.-C. Liang, S. Sun, and C. K. Ho, "Block-iterative generalized decision feedback equalizers for large MIMO systems: Algorithm design and asymptotic performance analysis," IEEE Trans. Signal Process., vol. 54, no. 6, pp. 2035-2048, Jun. 2006

[64] H. Zhao, H. Long, and W. Wang, "Tabu search detection for MIMO systems," in Proc. IEEE Int. Symp. PIMRC, Athens, Greece, Sep. 2007, pp. $1-5$.

[65] S. Mohammed and E. Larsson, "Per-antenna constant envelope precoding for large multi-user MIMO systems," IEEE Trans. Commun., vol. 61 , no. 3, pp. 1059-1071, Mar. 2013

[66] C. Peel, B. Hochwald, and A. Swindlehurst, "A vector-perturbation technique for near-capacity multiantenna multiuser communication-Part I: Channel inversion and regularization," IEEE Trans. Commun., vol. 53, no. 1, pp. 195-202, Jan. 2005.

[67] S. Wagner, R. Couillet, M. Debbah, and D. T. M. Slock, "Large system analysis of linear precoding in correlated MISO broadcast channels under limited feedback," IEEE Trans. Inf. Theory, vol. 58, no. 7, pp. 4509-4537, Jul. 2012

[68] C. Masouros, "Correlation rotation linear precoding for MIMO broadcast communications," IEEE Trans. Signal Process., vol. 59, no. 1, pp. 252-262, Jan. 2011.

[69] H. Q. Ngo, T. Duong, and E. Larsson, "Uplink performance analysis of multicell MU-MIMO with zero-forcing receivers and perfect CSI,' in Proc. IEEE Swedish Commun. Tech. Workshop, Stockholm, Sweden, Oct. 2011, pp. 40-45.

[70] H. Ngo, E. Larsson, and T. Marzetta, "The multicell multiuser MIMO uplink with very large antenna arrays and a finite-dimensional channel," IEEE Trans. Commun., vol. 61, no. 6, pp. 2350-2361, Jun. 2013. 
[71] A. Hu, T. Lv, H. Gao, Y. Lu, and E. Liu, "Pilot design for large-scale multi-cell multiuser MIMO systems," in Proc. IEEE Int. Conf. Commun., Budapest, Hungary, Jun. 2013, pp. 5381-5385.

[72] H. Wang, Y. Huang, S. Jin, F. Yu, and L. Yang, "Performance analysis on precoding and pilot scheduling in very large MIMO multi-cell systems," in Proc. IEEE Wireless Commun. Netw. Conf., Shanghai, China, Apr. 2013, pp. 2722-2726.

[73] H. Yin, D. Gesbert, M. C. Filippou, and Y. Liu, "Decontaminating pilots in massive MIMO systems," in Proc. IEEE ICC, Budapest, Hungary, Jun. 2013, pp. 3170-3175.

[74] F. Fernandes, A. Ashikhmin, and T. Marzetta, "Inter-cell interference in noncooperative TDD large scale antenna systems," IEEE J. Sel. Areas Commun., vol. 31, no. 2, pp. 192-201, Feb. 2013.

[75] F. Fabio, A. Alexei, and M. Thomas, "Interference reduction on cellular networks with large antenna arrays," in Proc. IEEE ICC, Ottawa, ON, Canada, May 2012, pp. 5773-5775

[76] H. Q. Ngo and E. Larsson, "EVD-based channel estimation in multicell multiuser MIMO systems with very large antenna arrays," in Proc. IEEE ICASSP, Kyoto, Japan, May 2012, pp. 3249-3252.

[77] R. R. Müller, M. Vehkaperä, and L. Cottatellucci, "Blind pilot decontamination," in Proc.17th ITG Workshop Smart Antennas, Stuttgart, Germany, Mar. 2013, pp. 1-6.

[78] A. Ashikhmin and T. Marzetta, "Pilot contamination precoding in multicell large scale antenna systems," in Proc. ISIT, Cambridge, MA, USA, Jul. 2012, pp. 1137-1141.

[79] J. Jose, A. Ashikhmin, T. Marzetta, and S. Vishwanath, "Pilot contamination and precoding in multi-cell TDD systems," IEEE Trans. Wireless Commun., vol. 10, no. 8, pp. 2640-2651, Jun. 2011.

[80] K. Truong and R. Heath, "Effects of channel aging in massive MIMO systems," J. Commun. Netw., vol. 15, no. 4, pp. 338-351, Sep. 2013.

[81] P. Taluja and B. Hughes, "Diversity limits of compact broadband multi-antenna systems," IEEE J. Sel. Areas. Commun., vol. 31, no. 2, pp. 326-337, Jan. 2013.

[82] A. Pitarokoilis, S. K. Mohammed, and E. G. Larsson, "Uplink performance of time-reversal MRC in massive MIMO systems subject to phase noise," IEEE Trans. Wireless Commun., vol. 15, no. 99, pp. 1-1, Nov. 2014

[83] E. Björnson, J. Hoydis, M. Kountouris, and M. Debbah, "Massive MIMO systems with non-ideal hardware: Energy efficiency, estimation, and capacity limits," IEEE Trans. Inf. Theory, vol. 60, no. 11, pp. 7112-7139, Nov. 2014.

[84] X. Rao and V. Lau, "Distributed compressive CSIT estimation and feedback for FDD multi-user massive MIMO systems," IEEE Trans. Signal Process., vol. 62, no. 12, pp. 3261-3271, Jun. 2014.

[85] Y. Ma, D. Zhang, A. Leith, and Z. Wang, "Error performance of transmit beamforming with delayed and limited feedback," IEEE Trans. Wireless Commun., vol. 8, no. 3, pp. 1164-1170, Mar. 2009.

[86] J. Zhang, M. Kountouris, J. Andrews, and R. Heath, "Multi-mode transmission for the MIMO broadcast channel with imperfect channel state information," IEEE Trans. Wireless Commun., vol. 59, no. 3, pp. 803-814, Mar. 2011.

[87] T. D. Novlan, R. K. Ganti, A. Ghosh, and J. G. Andrews, "Analytical evaluation of fractional frequency reuse for OFDMA cellular networks," IEEE Trans. Wireless Commun., vol. 10, no. 12, pp. 4294-4305, Oct. 2011.

[88] X. Mao, A. Maaref, and K. H. Teo, "Adaptive soft frequency reuse for inter-cell interference coordination in SC-FDMA based 3GPP LTE uplinks," in Proc. IEEE GLOBECOM, New Orleans, LO, USA, Nov. 2008, pp. 1-6.

[89] A. Adhikary, E. Al Safadi, and G. Caire, "Massive MIMO and intertier interference coordination," in Proc. ITA Workshop, San Diego, CA, USA, Feb. 2014, pp. 1-10.

[90] S. Ramprashad and G. Caire, "Cellular vs. network MIMO: A comparison including the channel state information overhead," in Proc. IEEE Int. Symp. PIMRC, Tokyo, Japan, Sep. 2009, pp. $878-884$.

[91] K. Hosseini, W. Yu, and R. Adve, "Large-scale MIMO versus network MIMO for multicell interference mitigation," IEEE J. Sel. Topics Signal Process., vol. 8, no. 5, pp. 930-941, Oct. 2014.

[92] C.-S. Lee, M.-C. Lee, C.-J. Huang, and T.-S. Lee, "Sectorization with beam pattern design using 3D beamforming techniques," in Proc. APSIPA Conf., Kaohsiung, Taiwan, Oct. 2013, pp. $1-5$.

[93] H. Halbauer, S. Saur, J. Koppenborg, and C. Hoek, "Interference avoidance with dynamic vertical beamsteering in real deployments," in Proc. IEEE WCNCW, Paris, France, Apr. 2012, pp. 294-299.
[94] V. D'Amico et al., "Advanced interference management in ARTIST4G: Interference avoidance," in Proc. EUWIT, Paris, France, Sep. 2010, pp. 21-24.

[95] M. Kountouris and N. Pappas, "HetNets and massive MIMO: Modeling, potential gains, and performance analysis," in Proc. IEEE-APS Top. Conf. Wireless Commun., Torino, Italy, Sep. 2013, pp. 1319-1322.

[96] J. Hoydis, K. Hosseini, S. ten Brink, and M. Debbah, "Making smart use of excess antennas: Massive MIMO, small cells, and TDD," Bell Labs Tech. J., vol. 18, no. 2, pp. 5-21, Aug. 2013.

[97] K. Hosseini, J. Hoydis, S. ten Brink, and M. Debbah, "Massive MIMO and small cells: How to densify heterogeneous networks," in Proc. IEEE ICC, Budapest, Hungary, Jun. 2013, pp. 5442-5447.

[98] F. Capozzi, G. Piro, L. Grieco, G. Boggia, and P. Camarda, "Downlink packet scheduling in LTE cellular networks: Key design issues and a survey," IEEE Commun. Surveys Tuts., vol. 15, no. 2, pp. 678-700, 2nd Quart. 2013.

[99] E. Bjornson, M. Kountouris, and M. Debbah, "Massive MIMO and small cells: Improving energy efficiency by optimal soft-cell coordination," in Proc. IEEE ICC, Casablanca, Morocco, Jun. 2013, pp. $1-5$.

[100] H. Papadopoulos, G. Caire, and S. A. Ramprashad, "Achieving large spectral efficiencies from MU-MIMO with tens of antennas: Locationadaptive TDD MU-MIMO design and user scheduling," in Proc. Asilomar Conf. Signals, Syst., Comput., Pacific Grove, CA, USA, Nov. 2010, pp. 636-643.

[101] D. Ng, E. Lo, and R. Schober, "Energy-efficient resource allocation in OFDMA systems with large numbers of base station antennas," IEEE Trans. Wireless Commun., vol. 11, no. 9, pp. 3292-3304, Jul. 2012.

[102] J. Nam, A. Adhikary, J.-Y. Ahn, and G. Caire, "Joint spatial division and multiplexing: Opportunistic beamforming, user grouping and simplified downlink scheduling," IEEE J. Sel. Topics Signal Process., vol. 8, no. 5, pp. 876-890, Oct. 2014

[103] H. Huh, A. M. Tulino, and G. Caire, "Network MIMO with linear zeroforcing beamforming: Large system analysis, impact of channel estimation, and reduced-complexity scheduling," IEEE Trans. Inf. Theory, vol. 58, no. 5, pp. 2911-2934, May 2012.

[104] Y. Xu, G. Yue, and S. Mao, "User grouping for massive MIMO in FDD systems: New design methods and analysis," IEEE Access, vol. 2, pp. 947-959, Aug. 2014.

[105] P. de Kerret and D. Gesbert, "Degrees of freedom of the network MIMO channel with distributed CSI," IEEE Trans. Inf. Theory, vol. 58, no. 11, pp. 6806-6824, Nov. 2012.

[106] Y. Yang, B. Bai, W. Chen, and L. Hanzo, "A low-complexity cross-layer algorithm for coordinated downlink scheduling and robust beamforming under a limited feedback constraint," IEEE Trans. Veh. Technol., vol. 63, no. 1, pp. 107-118, Jan. 2014

[107] A. Liu and V. Lau, "Hierarchical interference mitigation for massive MIMO cellular networks," IEEE Trans. Signal Process., vol. 62, no. 18, pp. 4786-4797, Sep. 2014.

[108] R. Zakhour and S. Hanly, "Min-max fair coordinated beamforming via large systems analysis," in Proc. 8th ISWCS, Aachen, Germany, Nov. 2011, pp. 1990-1994.

[109] Y. Huang, C. W. Tan, and B. D. Rao, "Multicell network duality with instantaneous and statistical channel information: A nonlinear Perron-Frobenius characterization," in Proc. CISS, Princeton, NJ, USA, Mar. 2012, pp. 1-6.

[110] R. Zakhour and S. Hanly, "Min-max power allocation in cellular networks with coordinated beamforming," IEEE J. Sel. Areas Commun., vol. 31, no. 2, pp. 287-302, Feb. 2013.

[111] Y. Huang, C. W. Tan, and B. Rao, "Joint beamforming and power control in coordinated multicell: Max-min duality, effective network and large system transition," IEEE Trans. Wireless Commun., vol. 12, no. 6, pp. 2730-2742, Jun. 2013.

[112] A. Swindlehurst, E. Ayanoglu, P. Heydari, and F. Capolino, "Millimeterwave massive MIMO: The next wireless revolution?," IEEE Commun Mag., vol. 52, no. 9, pp. 56-62, Sep. 2014.

[113] A. Sulyman et al., "Radio propagation path loss models for $5 \mathrm{G}$ cellular networks in the $28 \mathrm{GHz}$ and $38 \mathrm{GHz}$ millimeter-wave bands," IEEE Commun. Mag., vol. 52, no. 9, pp. 78-86, Sep. 2014.

[114] T. Bai, A. Alkhateeb, and R. Heath, "Coverage and capacity of millimeter-wave cellular networks," IEEE Commun. Mag., vol. 52, no. 9, pp. 70-77, Sep. 2014.

[115] W. Hong, K.-H. Baek, Y. Lee, Y. Kim, and S.-T. Ko, "Study and prototyping of practically large-scale mmWave antenna systems for 5G cellular devices," IEEE Commun. Mag., vol. 52, no. 9, pp. 63-69, Sep. 2014 
[116] C. Dehos, J. González, A. De Domenico, D. Kténas, and L. Dussopt, "Millimeter-wave access and backhauling: the solution to the exponential data traffic increase in $5 \mathrm{G}$ mobile communications systems?" IEEE Commun. Mag., vol. 52, no. 9, pp. 88-95, Sep. 2014.

[117] H. Q. Ngo, H. Suraweera, M. Matthaiou, and E. Larsson, "Multipair fullduplex relaying with massive arrays and linear processing," IEEE J. Sel. Topics Signal Process., vol. 32, no. 9, pp. 1721-1737, Sep. 2014.

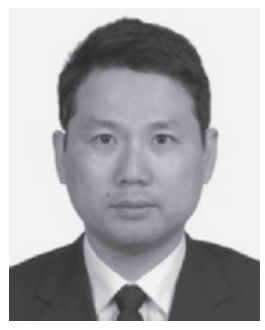

Kan Zheng received the B.S., M.S., and Ph.D. degrees from the Beijing University of Posts and Telecommunications (BUPT), Beijing, China, in 1996, 2000, and 2005, respectively. He is currently a Professor at BUPT. He is the author of more than 200 journal articles and conference papers in the field of resource optimization in wireless networks, M2M networks, VANET, and so on. He has rich industry experiences on the standardization of the new emerging technologies. Dr. Zheng holds editorial board positions for several journals. He has organized several special issues in famous journals, including the IEEE COMMUNICATIONS ON SURVEYS AND TUTORIALS and Transactions on Emerging Telecommunications Technologies.

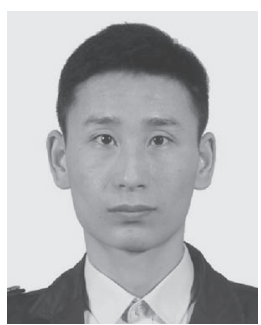

and signal processing.
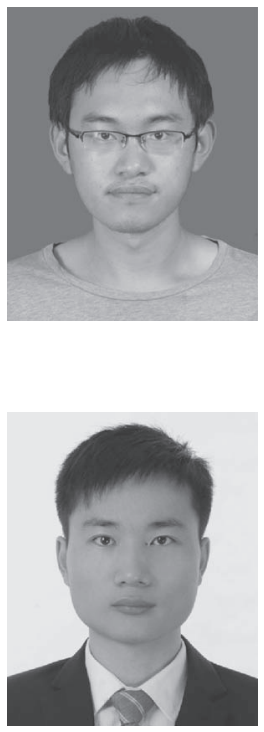

Long Zhao received the B.S. degree from Nanjing University of Posts and Telecommunications, Nanjing, China, in 2008 and the M.S. degree from Harbin Engineering University, Harbin, China, in 2011. He is currently working toward the Ph.D. degree with the Beijing University of Posts and Telecommunications, Beijing. From April 2014 to March 2015, he was a Visiting Scholar at the Department of Electrical Engineering, Columbia University, supervised by Prof. X. Wang. His research interests include wireless communications

Jie Mei received the B.S. degree from Nanjing University of Posts and Telecommunications, Nanjing, China, in 2013. He is currently working toward the Ph.D. degree at the Beijing University of Posts and Telecommunications, Beijing, China. His research interests include massive MIMO, millimeterwave mobile communications, and heterogeneous networks.

Bin Shao received the B.S. and M.S. degrees in communication engineering from the Beijing University of Posts and Telecommunications (BUPT), Beijing, China, in 2012 and 2015, respectively. He is currently with Realsil Microelectronics Corporation, Suzhou, China. His research interests include interference management, MIMO, and short-range wireless communication.

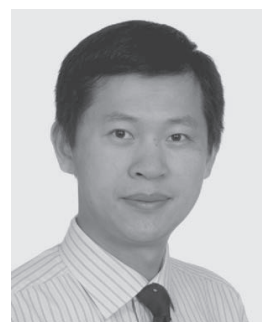

Wei Xiang (S'00-M'04-SM'10) received the B.Eng. and M.Eng. degrees in electronic engineering from the University of Electronic Science and Technology of China, Chengdu, China, in 1997 and 2000, respectively, and the Ph.D. degree in telecommunications engineering from the University of South Australia, Adelaide, Australia, in 2004.

Since January 2004, he has been with the School of Mechanical and Electrical Engineering, University of Southern Queensland, Toowoomba, Australia, where he currently holds a faculty post of Associate Professor. His research interests are in the broad area of communications and information theory, particularly coding and signal processing for multimedia communications systems. Dr. Xiang is an Editor of the IEEE COMMUNICATIONS LETTERS. He has been awarded a number of prestigious fellowship titles, including the Queensland International Fellow (2010-2011) by the Queensland Government of Australia, the Endeavour Research Fellow (2012-2013) by the Commonwealth Government of Australia, the Smart Futures Fellow (2012-2015) by the Queensland Government of Australia, and the JSPS Invitational Fellow (2014-2015) by the Japan Society for the Promotion of Science (JSPS). He was a recipient of the Best Paper Award at 2011 IEEE WCNC and the USQ Excellence in Research Award in 2013.

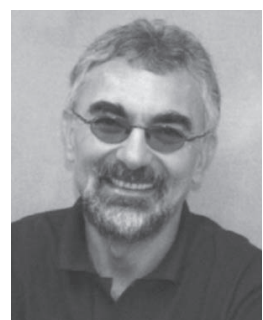

Lajos Hanzo received the M.S. degree in electronics and the Ph.D. degree from the Technical University of Budapest, Budapest, Hungary, in 1976 and 1983, respectively; the D.Sc. degree from the University of Southampton, Southampton, U.K., in 2004; and the "Doctor Honoris Causa" degree from the Technical University of Budapest in 2009. During his 38-year career in telecommunications, he has held various research and academic posts in Hungary, Germany, and the U.K. Since 1986, he has been with the School of Electronics and Computer Science, University of Southampton, where he holds the Chair in Telecommunications. He is currently directing an academic research team, working on a range of research projects in the field of wireless multimedia communications sponsored by industry, the Engineering and Physical Sciences Research Council UK, the European IST Programme, and the Mobile Virtual Centre of Excellence, UK. During 2008-2012, he was a Chaired Professor at Tsinghua University, Beijing. He is an enthusiastic supporter of industrial and academic liaison and offers a range of industrial courses. He has successfully supervised about $100 \mathrm{Ph} . \mathrm{D}$. students, coauthored 20 John Wiley/IEEE Press books on mobile radio communications totaling in excess of 10000 pages, and published more than 1400 research entries at IEEE Xplore. Dr. Hanzo is a Fellow of the Royal Academy of Engineering, the Institution of Engineering and Technology, and the European Association for Signal Processing. He is also a Governor of the IEEE Vehicular Technology Society. During 2008-2012, he was the Editor-in-Chief of IEEE Press. He has served as the Technical Program Committee Chair and the General Chair of IEEE conferences, has presented keynote lectures, and has been awarded a number of distinctions. For further information on research in progress and associated publications, please refer to http://wwwmobile.ecs.soton.ac.uk. 